Documents de travail du Département des Affaires économiques de l'OCDE No. 1478

Insertion de la Tunisie dans les chaines de valeur mondiales et role des entreprises offshore
Isabelle Joumard,

Souad Dhaoui, Hermes Morgavi 
Organisation de Coopération et de Développement Économiques

Organisation for Economic Co-operation and Development

25-Jun-2018

DEPARTEMENT DES AFFAIRES ECONOMIQUES

Français - Or. Français

\section{INSERTION DE LA TUNISIE DANS LES CHAINES DE VALEUR MONDIALES ET ROLE DES ENTREPRISES OFFSHORE}

\section{DOCUMENTS DE TRAVAIL DU DEPARTEMENT DES AFFAIRES ECONOMIQUES DE L'OCDE $\mathrm{N}^{\circ} 1478$}

Par Isabelle Joumard, Souad Dhaoui et Hermes Morgavi

Les documents de travail de l'OCDE ne doivent pas être présentés comme exprimant les vues officielles de l'OCDE ou de ses pays membres. Les opinions exprimées et les arguments employés sont ceux des auteur $(s)$.

Accord pour publication donné par Álvaro Pereira, Chef Economiste par intérim, Département des Affaires économiques.

Les Documents de travail du Département des Affaires économiques sont disponibles à www.oecd.org/eco/workingpapers.

JT03434070

Ce document, ainsi que les données et cartes qu'il peut comprendre, sont sans préjudice du statut de tout territoire, de la souveraineté s'exerçant sur ce dernier, du tracé des frontières et limites internationales, et du nom de tout territoire, ville ou région. 
Les documents de travail de l'OCDE ne doivent pas être présentés comme exprimant les vues officielles de l'OCDE ou de ses pays membres. Les opinions exprimées et les arguments employés sont ceux des auteur(s).

Les documents de travail exposent des résultats préliminaires ou des travaux de recherche en cours menés par l'auteur/les auteurs et sont publiés pour stimuler le débat sur un large éventail de questions sur lesquelles l'OCDE travaille.

Les commentaires sur les documents de travail sont les bienvenus et peuvent être adressés au Département des Affaires économiques, OCDE, 2 rue André-Pascal, 75775 Paris Cedex 16, France ou à l'adresse mél. suivante : eco.contact@oecd.org.

Les Documents de travail du Département des Affaires économiques sont disponibles à www.oecd.org/eco/workingpapers.

Les données statistiques concernant Israël sont fournies par et sous la responsabilité des autorités israéliennes compétentes. L'utilisation de ces données par l'OCDE est sans préjudice du statut des hauteurs du Golan, de Jérusalem Est et des colonies de peuplement israéliennes en Cisjordanie aux termes du droit international.

\section{(C) OCDE (2018)}

Vous êtes autorisés à copier, télécharger ou imprimer du contenu OCDE pour votre utilisation personnelle. Vous pouvez inclure des extraits des publications, des bases de données et produits multimédia de l'OCDE dans vos documents, présentations, blogs, sites Internet et matériel d'enseignement, sous réserve de faire mention de la source OCDE et du copyright. Les demandes pour usage commercial ou de traduction devront être adressées à rights@oecd.org. 
ECO/WKP(2018)26

\section{ABSTRACT/RÉSUMÉ}

\section{Insertion de la Tunisie dans les chaines de valeur mondiales et rôle des entreprises offshore}

L'ouverture de la Tunisie aux échanges internationaux et son intégration dans les chaines de valeur mondiales ont fortement progressé depuis le milieu des années 90, témoignant des avantages comparatifs du pays. Les exportations ont sensiblement augmenté, tirées par le secteur manufacturier, avec une transformation en faveur de secteurs plus intensifs en technologie et compétences. La montée en gamme et la diversification des exportations augurent d'un potentiel de croissance élevé. Cette bonne performance est pour l'essentiel le fait d'entreprises entièrement exportatrices (dites offshores), avec peu d'effet d'entrainement sur le reste de l'économie. En effet, ces entreprises s'approvisionnent peu sur le marché local et servent rarement la demande locale, reflétant en partie la complexité des procédures douanières, fiscales et administratives. La levée des contraintes à l'exportation rencontrées par les entreprises du secteur onshore et le décloisonnement entre régimes offshore et onshore permettraient à la Tunisie de se hisser dans les chaines de valeur mondiales et d'en tirer plus d'avantages, notamment en termes de progrès technologique, de création d'emplois et de richesse.

Classification JEL ; F1, F14, F15, F6, O55

Mots-clés: Tunisie, échanges commerciaux, chaines de valeur mondiales, performances à l'exportation, secteur offshore

$* * * * * *$

\section{Tunisia's inclusion in global value chains and the role of offshore companies}

Tunisia's trade Tunisia's openness and its integration into global value chains has improved significantly since the mid-1990s, reflecting the country's comparative advantages. Exports have surged, led by the manufacturing sector, with a shift towards more technology- and skills-intensive goods. The upscaling and diversification of exports should enable the Tunisian economy to grow fast. However, this good export performance is mainly due to exporting-only companies (the so-called offshore sector) with little trickle-down effect. Offshore companies rarely buy inputs from onshore companies and few of their outputs are sold on the domestic market, partly reflecting the complexity of customs, tax and administrative procedures. Lifting export constraints faced by onshore companies and lowering barriers between offshore and onshore companies would allow Tunisia to climb into global value chains and get more benefits, in particular faster technological progress, and more job and value added creation.

JEL codes: F1, F14, F15, F6, O55

Keywords: Tunisia, trade, global value chains, export performance, offshore sector 


\section{TABLE OF CONTENTS}

\section{INSERTION DE LA TUNISIE DANS LES CHAINES DE VALEUR MONDIALES ET ROLE DES ENTREPRISES OFFSHORE .............................................................................................}

Un positionnement favorable de la Tunisie dans les chaines de valeur mondiales...................................5

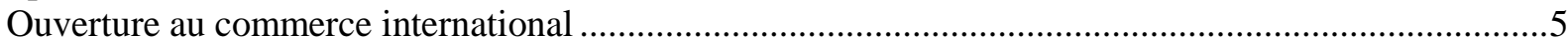

Exportations tunisiennes : augmentation, diversification et sophistication........................................6

Participation aux chaines de valeur mondiales mesurée par la valeur ajoutée .......................................10

Contribution du secteur offshore aux exportations, à l'emploi et au développement régional ..................14

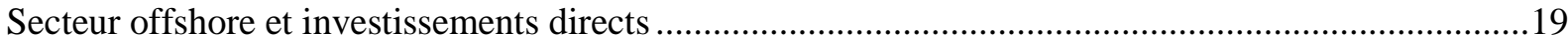

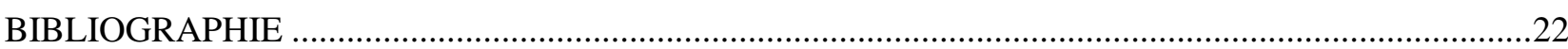

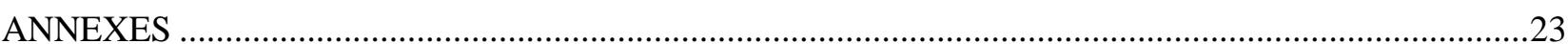

\section{Graphiques}

Graphique 1. Ouverture au commerce et nature des exportations....................................................6

Graphique 2. La structure des exportations se rapproche de celle des pays de l'OCDE.....................7

Graphique 3. Diversification et montée en gamme des exportations ..................................................

Graphique 4. Les exportations sont moins dépendantes d'un petit nombre de produits .......................9

Graphique 5. Classement des pays sur l'échelle de la complexité économique .................................10

Graphique 6. Insertion de la Tunisie dans les chaines de valeur mondiales......................................11

Graphique 7. Participation de la Tunisie dans les chaines de valeur mondiales par filières, $2011 \ldots . .12$

Graphique 8. Transition politique: des progrès considérables pour la démocratie..............................13

Graphique 9. Exportations et importations de marchandises par régime ........................................15

Graphique 10. Entreprises et emplois dans le secteur offshore.......................................................

Graphique 11. Répartition des entreprises offshore par région, 2016 …..........................................17

Graphique 12. Intensité de présence des entreprises offshore par gouvernorat .................................17

Graphique 13. Performance en logistique de transport et facilité à passer les biens en douane.............18

Graphique 14. Les flux et stocks d'investissements directs étrangers sont plutôt élevés .....................20

Graphique 15. Évolution de la structure des investissements directs de l'étranger..............................21

\section{Encadrés}

Encadré 1. Commerce de services et exportations de produits manufacturés

Encadré 2. Entreprises totalement exportatrices et régime "offshore": définition et rôle dans l'économie16 
ECO/WKP(2018)26

\title{
INSERTION DE LA TUNISIE DANS LES CHAINES DE VALEUR MONDIALES ET ROLE DES ENTREPRISES OFFSHORE
}

\author{
Isabelle Joumard, Souad Dhaoui et Hermes Morgavi ${ }^{1}$
}

1. L'ouverture de la Tunisie aux échanges internationaux et son intégration dans les chaines de valeur mondiales ont fortement progressé depuis le milieu des années 90 , témoignant des avantages comparatifs du pays. Les exportations ont sensiblement augmenté, tirées par le secteur manufacturier, avec une transformation en faveur de secteurs plus intensifs en technologie et compétences. L'analyse des échanges commerciaux sur la base de la valeur ajoutée remet aussi en cause la perception selon laquelle les activités à faible teneur en valeur ajoutée, tel l'assemblage, dominent. La montée en gamme et la diversification des exportations augurent d'un potentiel de croissance élevé.

2. Cette bonne performance est pour l'essentiel le fait d'entreprises entièrement exportatrices (dites offshores) bien intégrées dans les chaines de valeur mondiales (CVM). Leur contribution à la création d'emplois formels a augmenté. L'excédent commercial du secteur offshore augmente aussi. Néanmoins, ces entreprises sont pour l'essentiel localisées proches des ports, contribuant à la concentration géographique de l'activité économique. En outre, elles entretiennent peu de relations avec le reste de l'économie, reflétant en partie la complexité des procédures douanières, fiscales et administratives. De leur côté, les entreprises du secteur onshore sont pénalisées par des difficultés lors du passage en douane de leurs produits et des services logistiques peu performants. La levée des contraintes à l'exportation rencontrées par les entreprises du secteur onshore et le décloisonnement entre régimes offshore et onshore permettraient à la Tunisie de se hisser dans les CVM et d'en tirer plus d'avantages, notamment en termes de progrès technologique, de création d'emplois et de richesse.

\section{Un positionnement favorable de la Tunisie dans les chaines de valeur mondiales}

\section{Ouverture au commerce international}

3. Les travaux récents de l'OCDE suggèrent que les échanges commerciaux peuvent contribuer à l'amélioration du bien-être de la population (OCDE, 2017a). Ils améliorent le pouvoir d'achat des consommateurs en leur permettant d'accéder à une gamme plus étendue de biens et services souvent à moindre coût. Les ménages les plus pauvres sont souvent ceux qui en bénéficient le plus car ils consacrent une part plus élevée de leur revenu aux produits de grande consommation (Fajgelbaum et Kandhelwal, 2016). Par ailleurs, les échanges commerciaux génèrent des gains de productivité en facilitant l'accès des entreprises à une gamme d'intrants plus vaste, en favorisant la diffusion de la connaissance étrangère et en contribuant à l'élargissement des marchés. Dans la zone OCDE, une augmentation de l'ouverture commerciale (somme des exportations et importations de biens et services rapportée au PIB) de 1 point de pourcentage se traduirait par une croissance de la productivité multi-factorielle de $0.2 \%$ après 5 ans et $0.6 \%$ sur le long-terme (Egert et Gal, 2017).

1. Isabelle Joumard est économiste principale au Département d'économie de l'OCDE, responsable du desk Inde et Tunisie. Souad Dhaoui est économiste en chef à l'Institut Tunisien de la Compétitivité et des Études Quantitatives et était détachée à l'OCDE lors de la préparation de ce travail. Hermes Morgavi est statisticien au Département d'économie de l'OCDE. Les auteurs remercient Piritta Sorsa et Zouhair El Kadhi pour leurs suggestions, les autorités tunisiennes, en particulier la FIPA et l'INS pour la mise à disposition des données, ainsi que les responsables d'entreprises offshore et onshore qui ont bien voulu répondre à leurs questions. Ils restent responsables de toutes les erreurs et omissions contenues dans ce document. 
4. En Tunisie, l'ouverture commerciale a fortement augmenté sur les deux dernières décennies. L'assouplissement progressif des barrières tarifaires et non tarifaires, la signature d'accords de libreéchange et la création d'un régime attractif pour les entreprises entièrement exportatrices - dit régime offshore - ont joué un rôle important. La Tunisie a adhéré à l'Organisation mondiale du commerce en 1995. Elle a signé en 1996 un accord d'association avec l'Union européenne visant au démantèlement progressif des barrières tarifaires et non-tarifaires et a obtenu le statut de partenaire privilégié en 2012 ; les négociations autour de l'accord de libre-échange complet et approfondi (ALECA) sont aussi en cours. Avec la Turquie, un accord de libre-échange est signé en 2004 et appliqué entièrement en 2014. Le régime tarifaire a en outre été simplifié, avec une réduction du nombre de bandes tarifaires de 54 en 2003 à 3 en 2017. Au final, la part des exportations et des importations dans le PIB s'approchait en 2016 de la moyenne OCDE, pour s'établir à un niveau supérieur à de nombreux pays émergents (graphique 1.A).

\section{Graphique 1. Ouverture au commerce et nature des exportations}

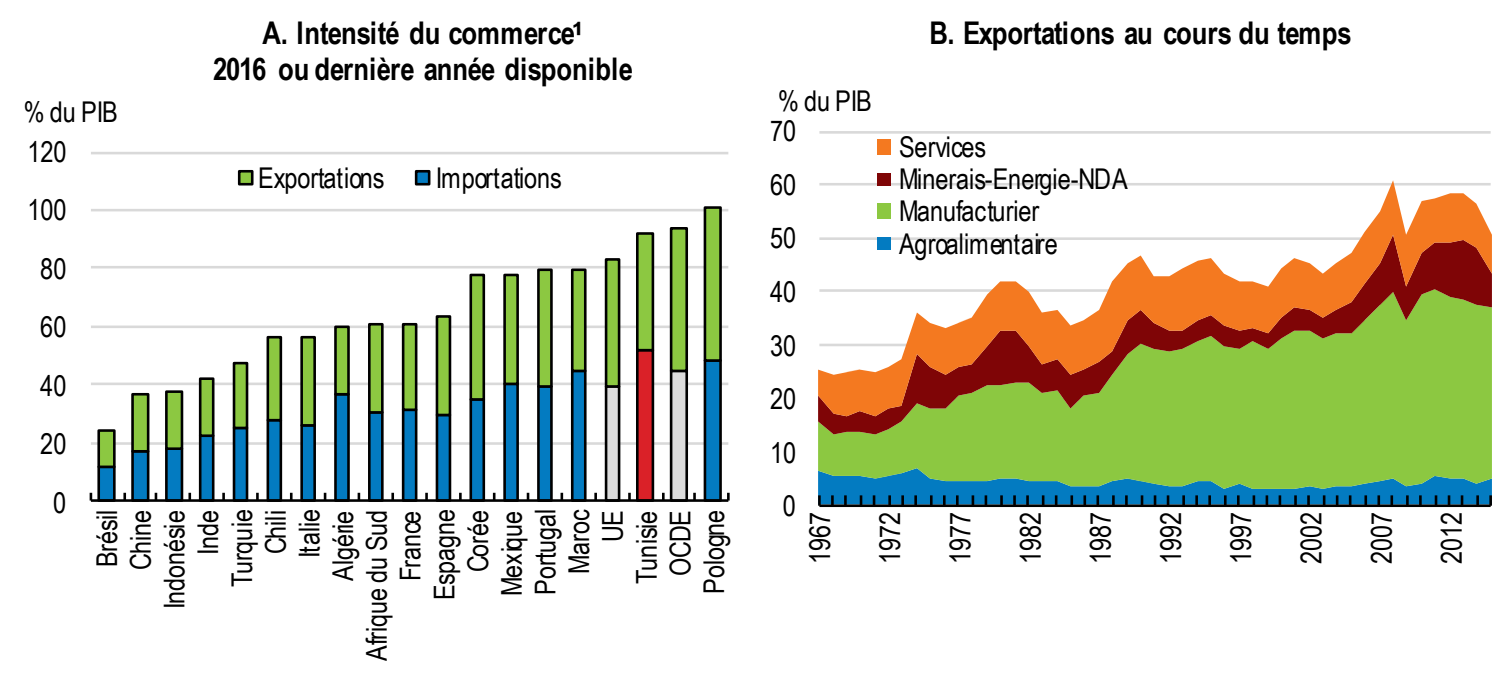

1. L'intensité du commerce est mesurée par la somme des importations et des exportations en \% du PIB.

Source: Institut national de la statistique; Banque mondiale, Indicateurs de développement mondial (WDI).

\section{Exportations tunisiennes : augmentation, diversification et sophistication}

5. Les exportations tunisiennes ont fortement progressé en volume et en qualité, tirées par le secteur manufacturier traditionnellement riche en emplois (graphique 1.B). Si les secteurs miniers et de l'énergie ont souffert d'une succession de mouvements sociaux depuis 2011, le secteur manufacturier a fait preuve d'une certaine résilience. Au final, la part des biens manufacturés dans les exportations totales a augmenté pour s'établir à $76 \%$, bien au-delà du niveau observé en Égypte, au Maroc et dans la plupart des autres pays de la région (graphique 2 .A). 
ECO/WKP(2018)26

\section{Graphique 2. La structure des exportations se rapproche de celle des pays de l'OCDE}

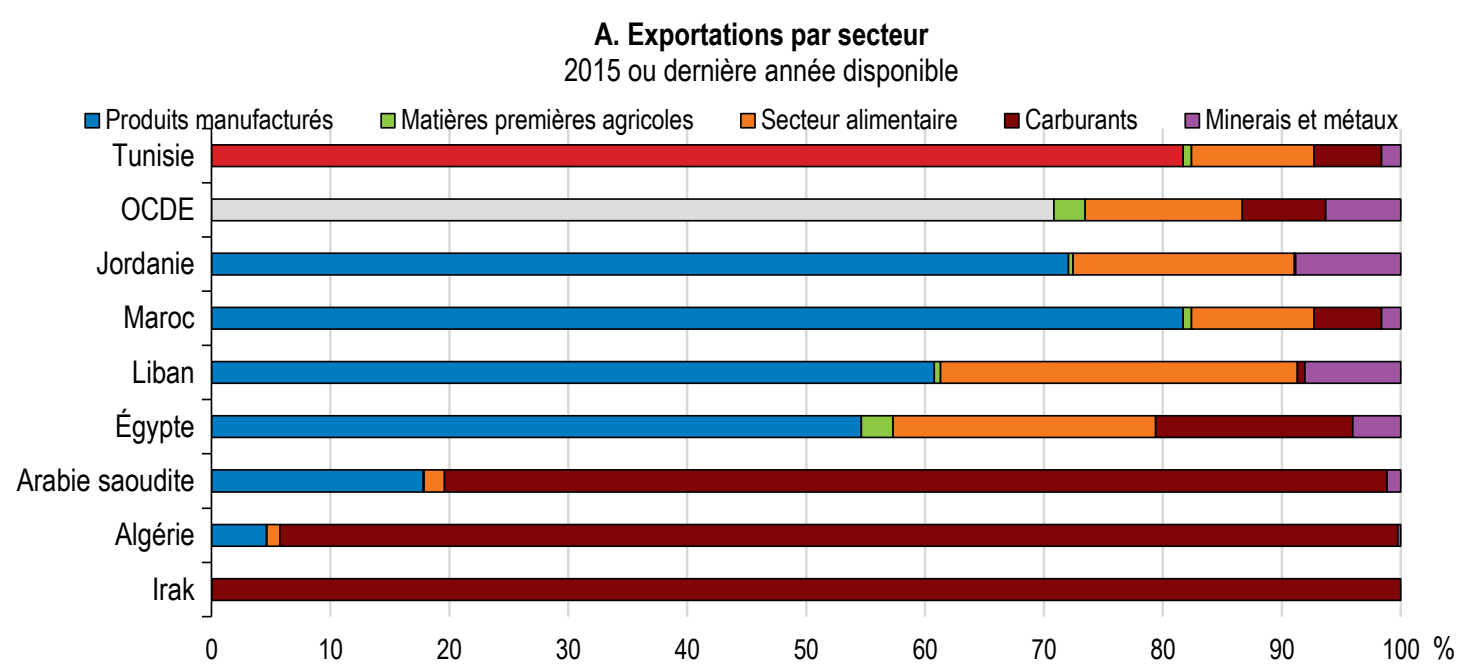

B. Structure des exportations par type d'utilisation

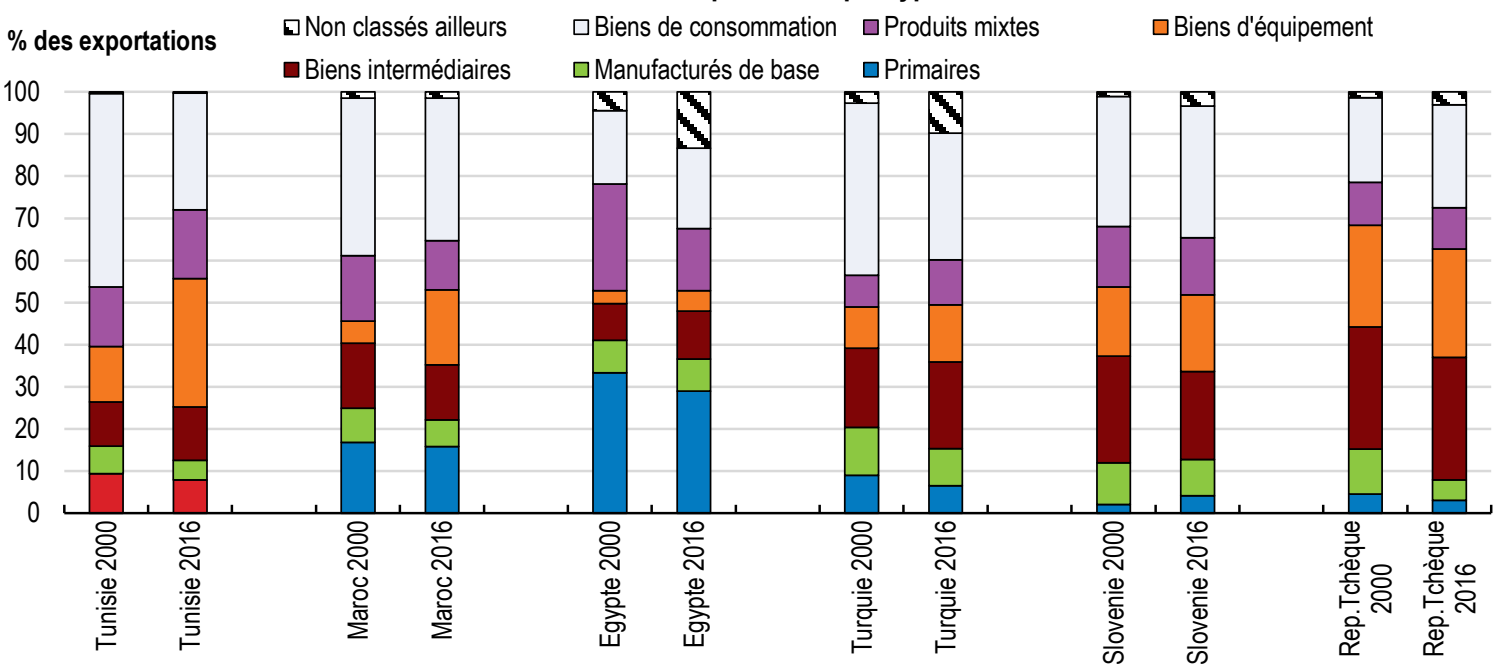

Source : Banque mondiale, Indicateurs de développement mondial (WDI) ; et Chelem database.

6. La structure des exportations manufacturières a connu une transformation significative, les industries mécaniques et électriques devenant le premier secteur exportateur. Cela se traduit aussi par une augmentation de la part des exportations des biens d'équipement et de biens intermédiaires. En matière d'exportation de biens d'équipement, l'une des composantes les plus dynamiques du commerce mondial de marchandises depuis le début des années 2000, la Tunisie se positionne mieux que nombreux pays émergents (graphique 2.B).

7. Les exportations tunisiennes se sont aussi diversifiées et ont monté en gamme. La Tunisie est le pays du Maghreb ayant le plus grand nombre de produits exportés jouissant d'un avantage comparatif révélé (graphique 3.A). La structure des exportations a aussi évolué au profit des produits intensifs en technologie et compétences (graphique 3.B). Les performances des filières électronique, mécanique et électrique, pharmaceutique et plastique sont particulièrement bonnes, reflétant un investissement de longue date dans le secteur éducatif, notamment les sciences et l'ingénierie. 
Graphique 3. Diversification et montée en gamme des exportations

A. Nombre des produits exportés avec avantage comparatif révélé, 2016

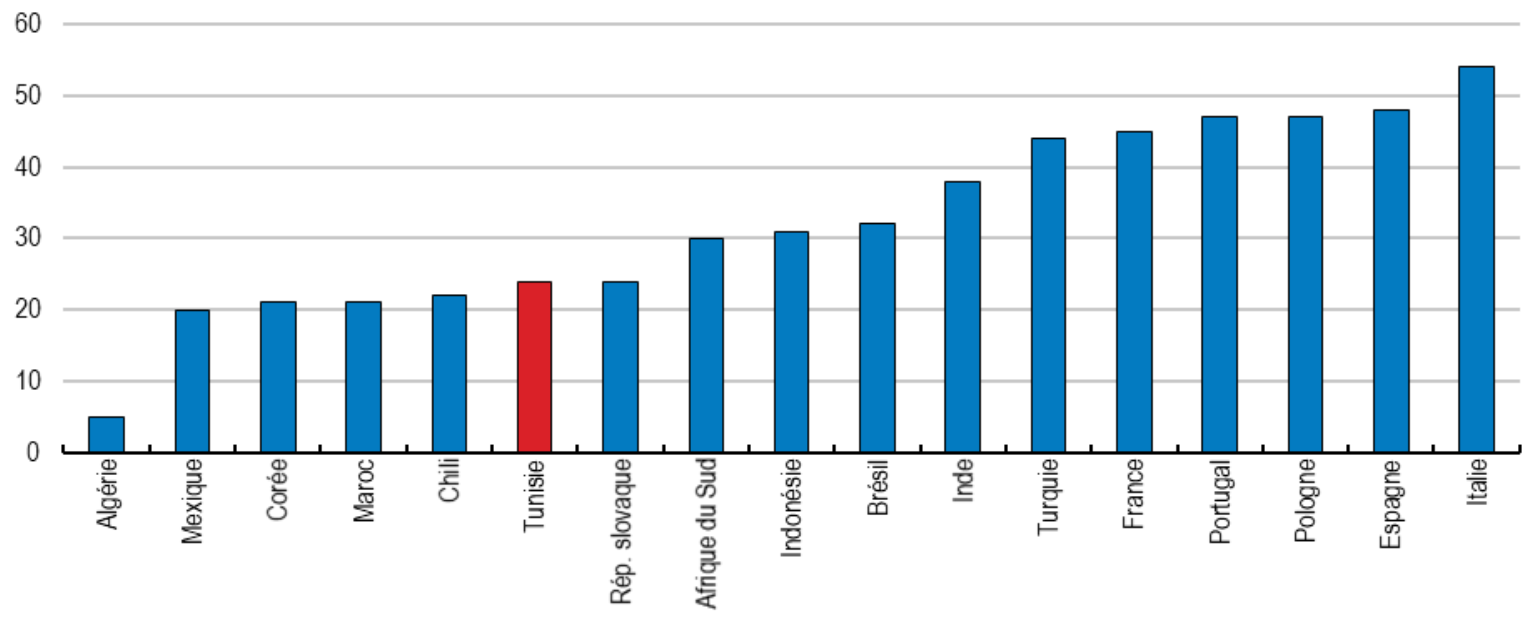

$\%$ des exportations $\quad$ B. L'intensité technologique des exportations manufacturières augmente manufacturières

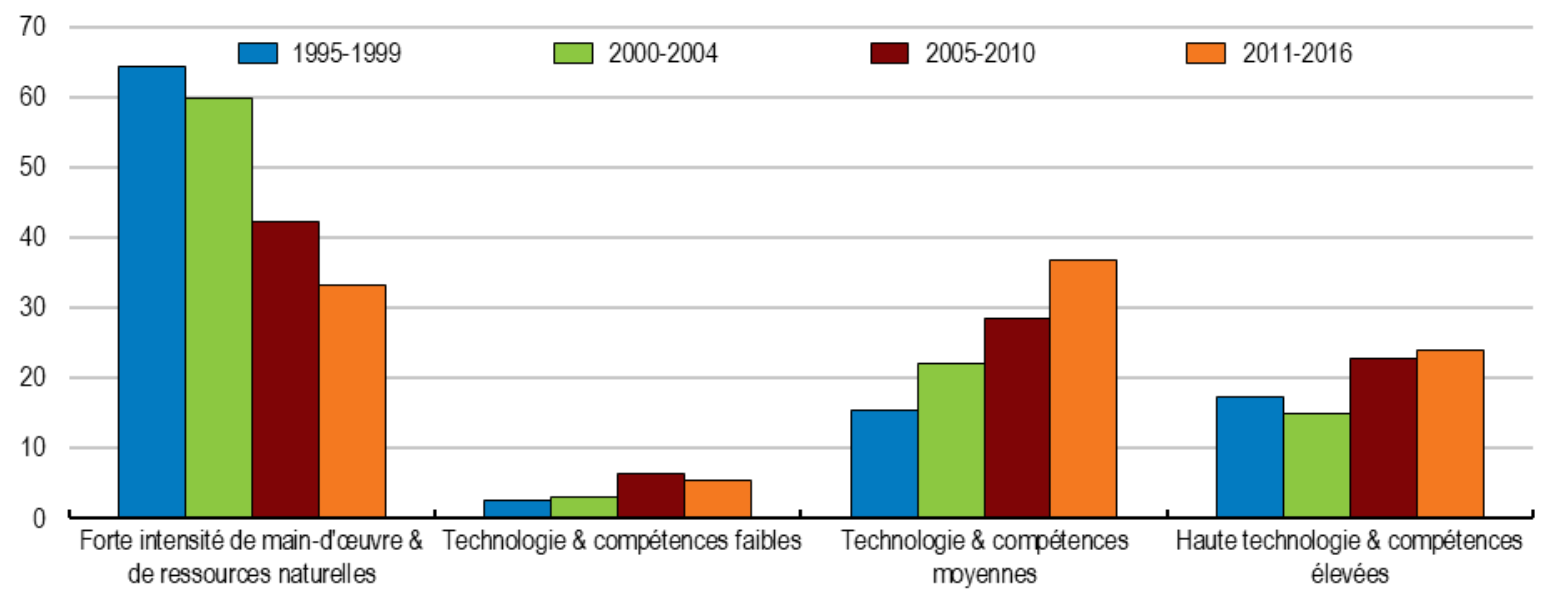

Note: La mesure de l'intensité technologique des exportations manufacturières est cohérente avec la définition de la CNUCED présentée dans le Rapport sur le Commerce et le Développement de 2002.

Source : Base de données Comtrade des Nations Unies ; calculs de l'OCDE ; et UNCTAD/STAT.

8. La structure des exportations par produits est peu concentrée par rapport aux autres pays de la région MENA (graphique 4). Ainsi, les quatre premiers produits exportés par la Tunisie représentaient $26 \%$ de ses exportations de biens en 2015, contre 33\% au Maroc et 56\% pour la moyenne des pays de la région MENA. Les progrès sur deux décennies ont été considérables. La sensibilité aux aléas de la conjoncture industrielle est, ainsi, réduite. Néanmoins, les exportations tunisiennes restent fortement concentrées sur un petit nombre de partenaires commerciaux - les quatre premiers partenaires (par ordre d'importance : France, Italie, Allemagne, Espagne) représentaient 61\% des exportations en 2015. 
Graphique 4. Les exportations sont moins dépendantes d'un petit nombre de produits

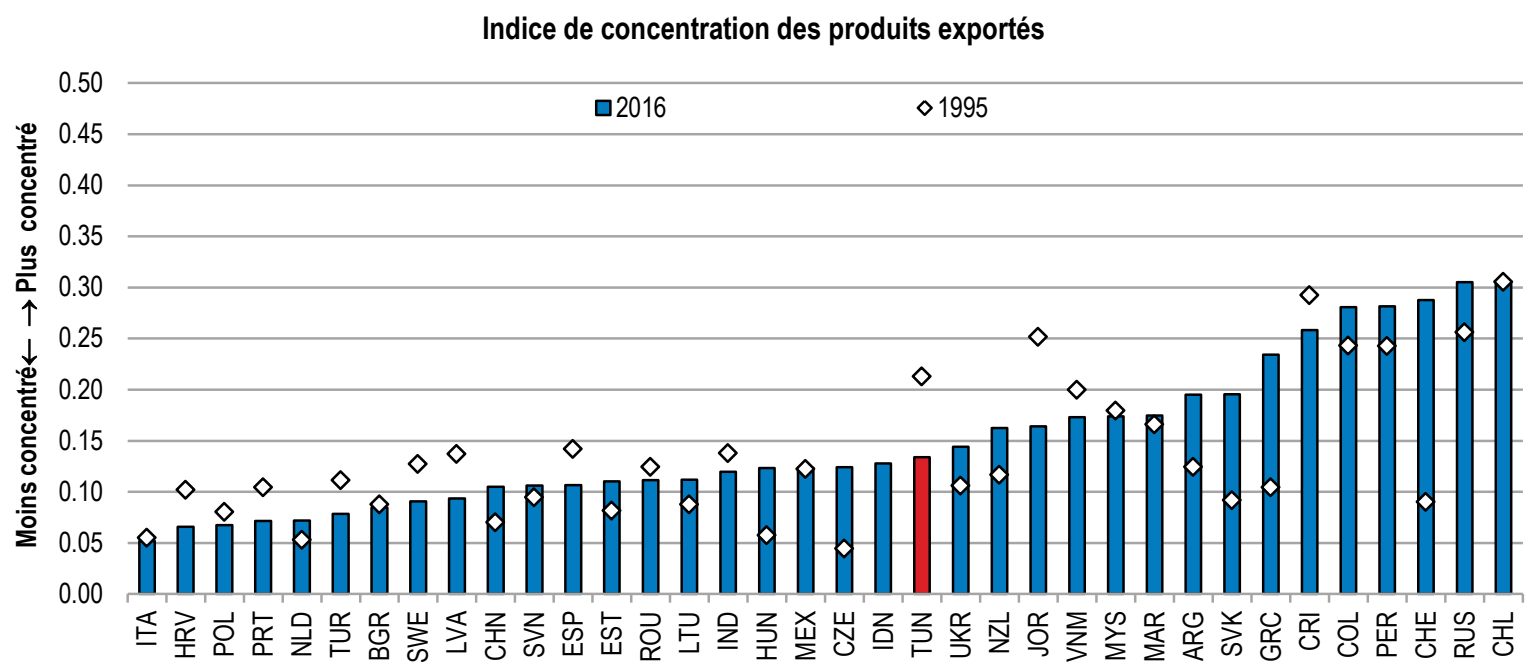

Note: une valeur de l'indice proche de 1 indique que les exportations du pays sont très concentrées sur quelques produits. Au contraire, des valeurs proches de 0 reflètent une répartition très homogène des parts de chaque produit.

Source: UNCTADstat.

9. Au final, la Tunisie a considérablement progressé sur l'échelle de la complexité économique, se rapprochant de la Turquie et de l'Inde (graphique 5). Ainsi, la Tunisie se plaçait en 2016 au $39^{\text {ème }}$ rang mondial sur l'échelle de la complexité qui reflète la sophistication, la diversification des exportations et la spécificité des exportations (Observatoire de la complexité économique, OEC-2017), devançant tous les pays d'Afrique. L'analyse par produit exporté révèle une forte progression de la Tunisie vers des produits plus complexes et sophistiqués, avec une densification du tissu industriel (Annexe 1). 


\section{Graphique 5. Classement des pays sur l'échelle de la complexité économique}
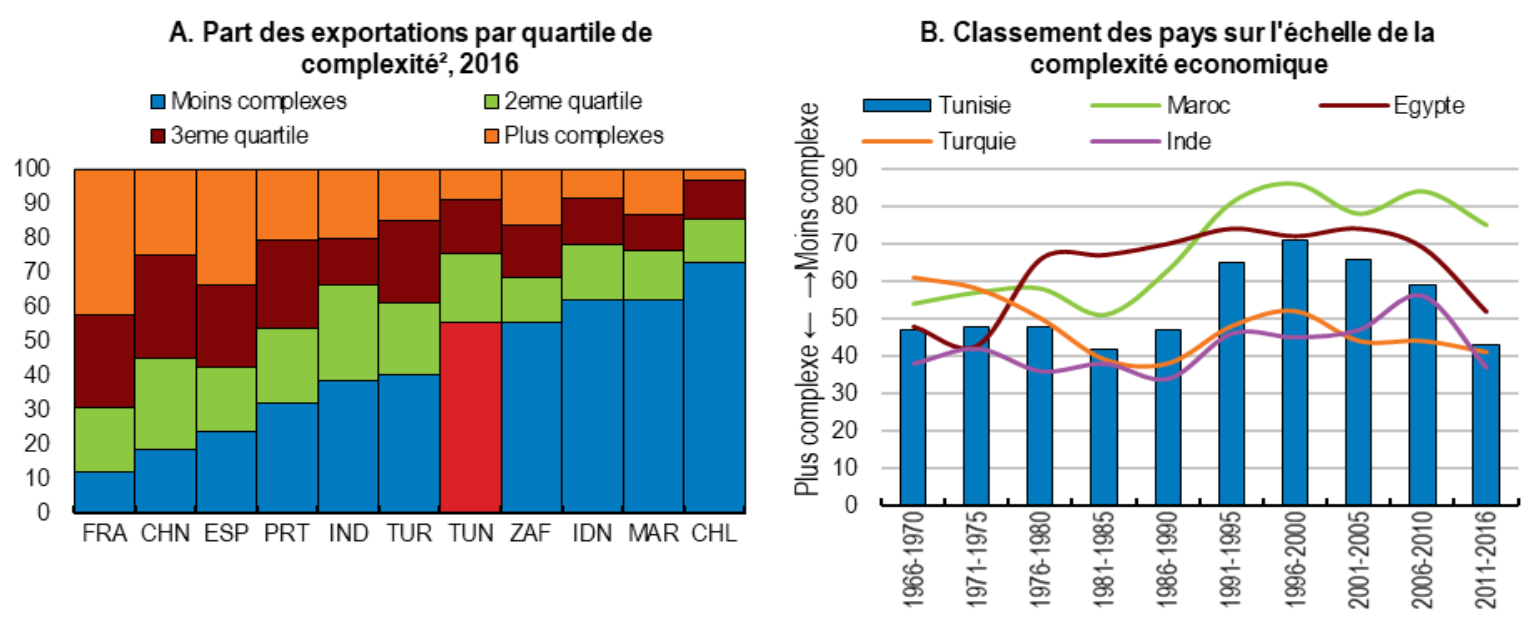

C. Projections de croissance en 2025 par région

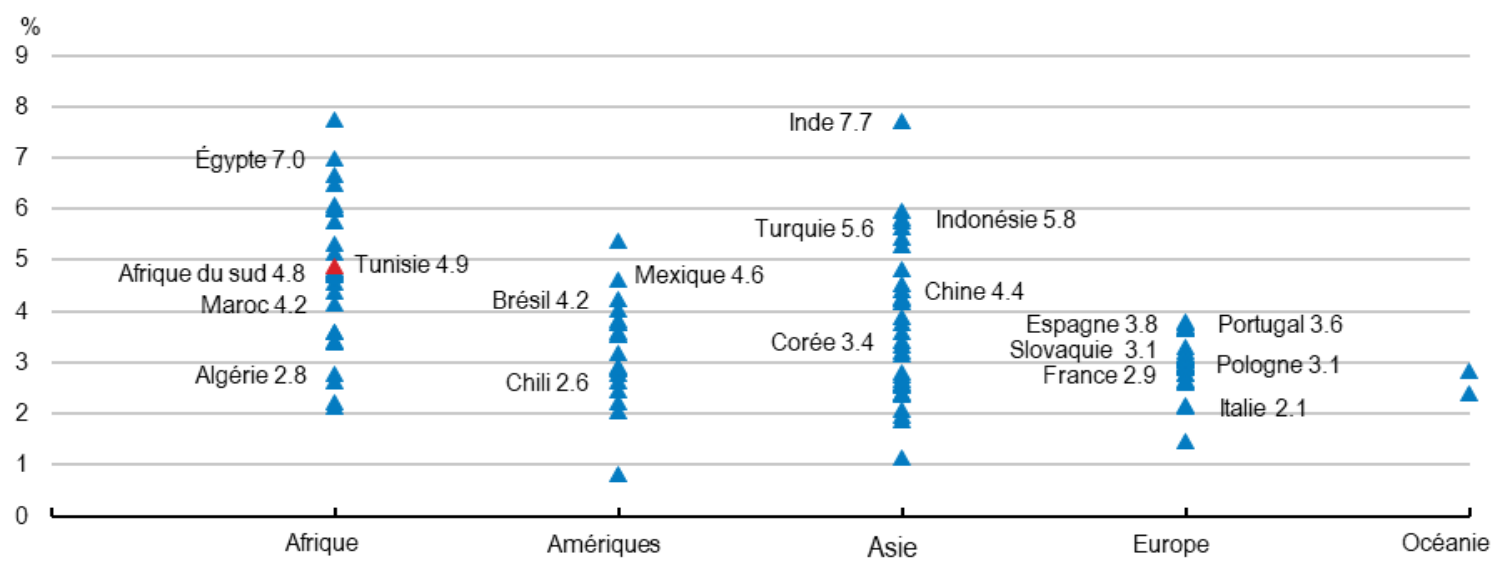

Note: L'indice de complexité économique est estimé à partir d'une mesure des connaissances disponibles dans un pays et qui se traduit par les produits qu'il fabrique. Il classe, ainsi, les économies en fonction de la complexité de leur panier d'exportations. Plus la complexité est élevée, plus le rang de classement du pays est faible, plus ses perspectives de croissance sont bonnes.

1. Le degré de complexité est défini comme dans Hausmann et al. (2007), "What you export matters", Journal of Economic Growth, Springer, vol. 12(1).

Source: Base de données Comtrade des Nations Unies ; calculs de l'OCDE ; Observatory of Economic Complexity (OEC); et The Atlas of Economic Complexity, 2016. Harvard Center for International Development.

10. Les perspectives de croissance d'ici à 2025 estimées sur la base de la complexité des exportations (The Atlas of Economic Complexity) sont très favorables. La structure du panier des produits exportés par un pays affecte la création de nouvelles lignes d'exportation et, en conséquence, son potentiel de croissance (Hausmann et Rodrik, 2003 ; Hausmann et al., 2007). Toutes choses égales par ailleurs, les pays qui se spécialisent dans les produits exportés par les pays ayant des niveaux de revenu par habitant élevés sont susceptibles d'améliorer leur croissance plus vite que ceux produisant les autres marchandises. Pour la Tunisie, le potentiel de croissance estimé sur la base de la composition des exportations approche 5\% (graphique 5.C).

\section{Participation aux chaines de valeur mondiales mesurée par la valeur ajoutée}

11. La base de données TiVA (Trade in Value Added) de l'OCDE et de l'OMC permet de mieux apprécier la participation aux chaines de valeur mondiales avec un accent sur la valeur ajoutée de chaque 
pays, allant ainsi au-delà de la valeur des flux commerciaux. La base de données TiVA permet aussi de mieux cerner le positionnement de chaque pays dans les chaines de valeur mondiales en identifiant, pour chaque pays, deux éléments: a) la part des intrants étrangers contenus dans les exportations du pays participation en amont ; b) la part des intrants produits par le pays contenus dans les exportations des autres pays - participation en aval.

12. La participation de la Tunisie dans les chaines de valeurs mondiales (CVM) a fortement progressé pour se situer à un niveau similaire à celle du Portugal et plus élevé que celle de nombreux pays émergents (graphique 6). Si la Tunisie importe une part croissante des intrants incorporés dans ses exportations, les biens et services produits en Tunisie sont aussi incorporés dans le processus de production de ses partenaires. C'est un positionnement prometteur, avec un accroissement des produits intensifs en technologies et qualifications moyennes et élevées.

\section{Graphique 6. Insertion de la Tunisie dans les chaines de valeur mondiales}

\section{A. Participation en amont et en aval des CVM (2011)}

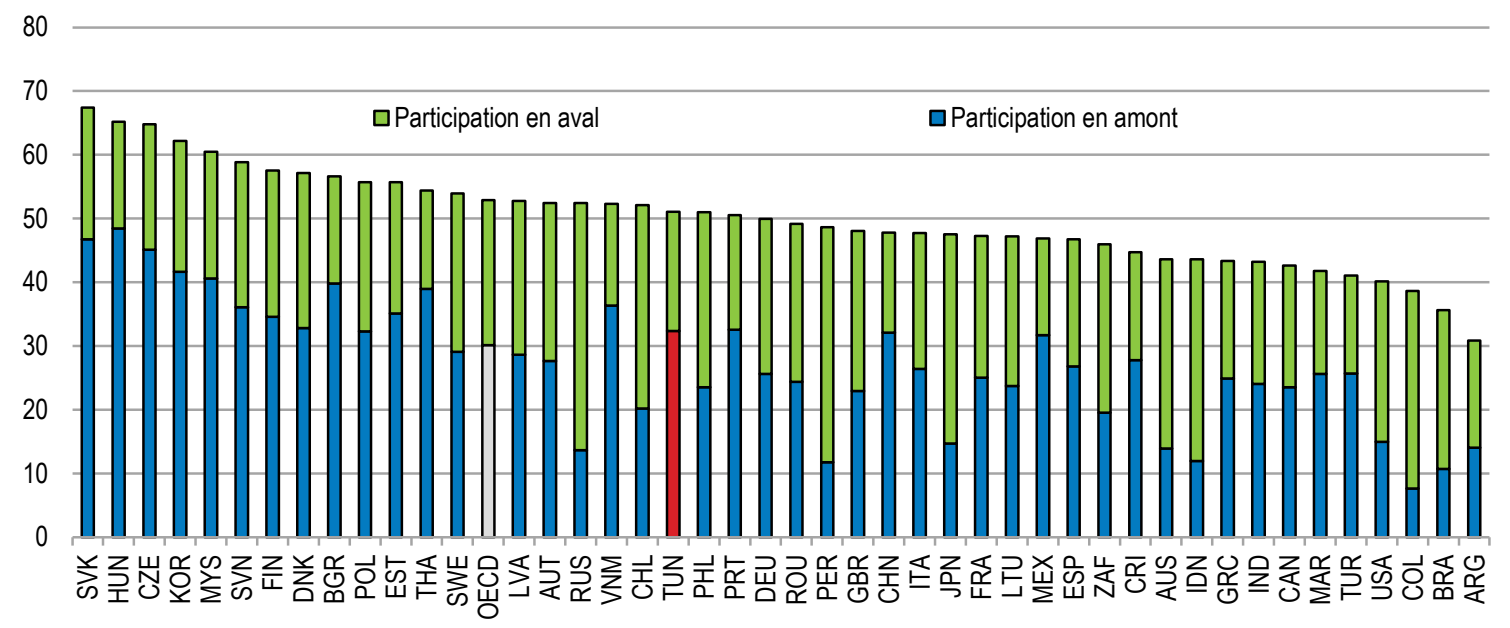

B. Changement du taux de participation aux CVM entre 2011 et 2005

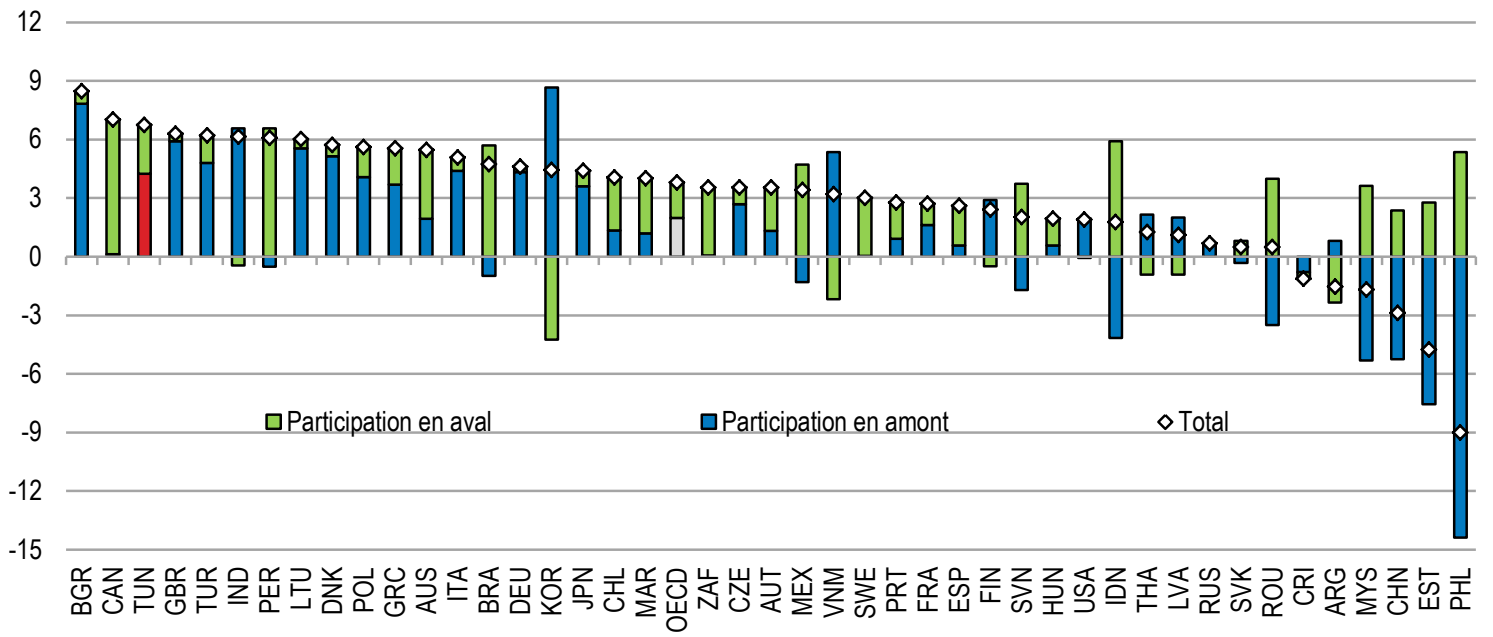

Note: La participation en aval correspond au contenu de la valeur ajoutée tunisienne dans les exportations des autres pays. La participation en amont correspond au contenu de la valeur ajoutée étrangère dans les exportations tunisiennes.

Source : Calculs des auteurs à partir de la base TiVA/ OCDE 
13. La Tunisie est particulièrement présente dans certaines chaines de valeur, notamment les produits électroniques, les industries mécaniques et électriques, le textile et l'habillement, la chimie, l'agroalimentaire et les services aux entreprises (graphique 7). Son taux de participation en aval - c.a.d. sa valeur ajoutée dans les exportations manufacturières des pays partenaires - est similaire à celui de l'Espagne. Il est supérieur à celui de l'Estonie, la Grèce, la Hongrie, le Portugal, la Turquie et de bien d'autres pays émergents, notamment le Maroc, remettant en cause la perception selon laquelle les activités d'assemblage dominent en Tunisie et que la valeur ajoutée est faible (graphique 1- annexe 2). Dans le secteur des services, la progression de la Tunisie concerne plus particulièrement les liens en amont - une part croissante de services étrangers est incorporée dans les exportations de la Tunisie (graphique 1- annexe 2).

\section{Graphique 7. Participation de la Tunisie dans les chaines de valeur mondiales par filières, 2011}

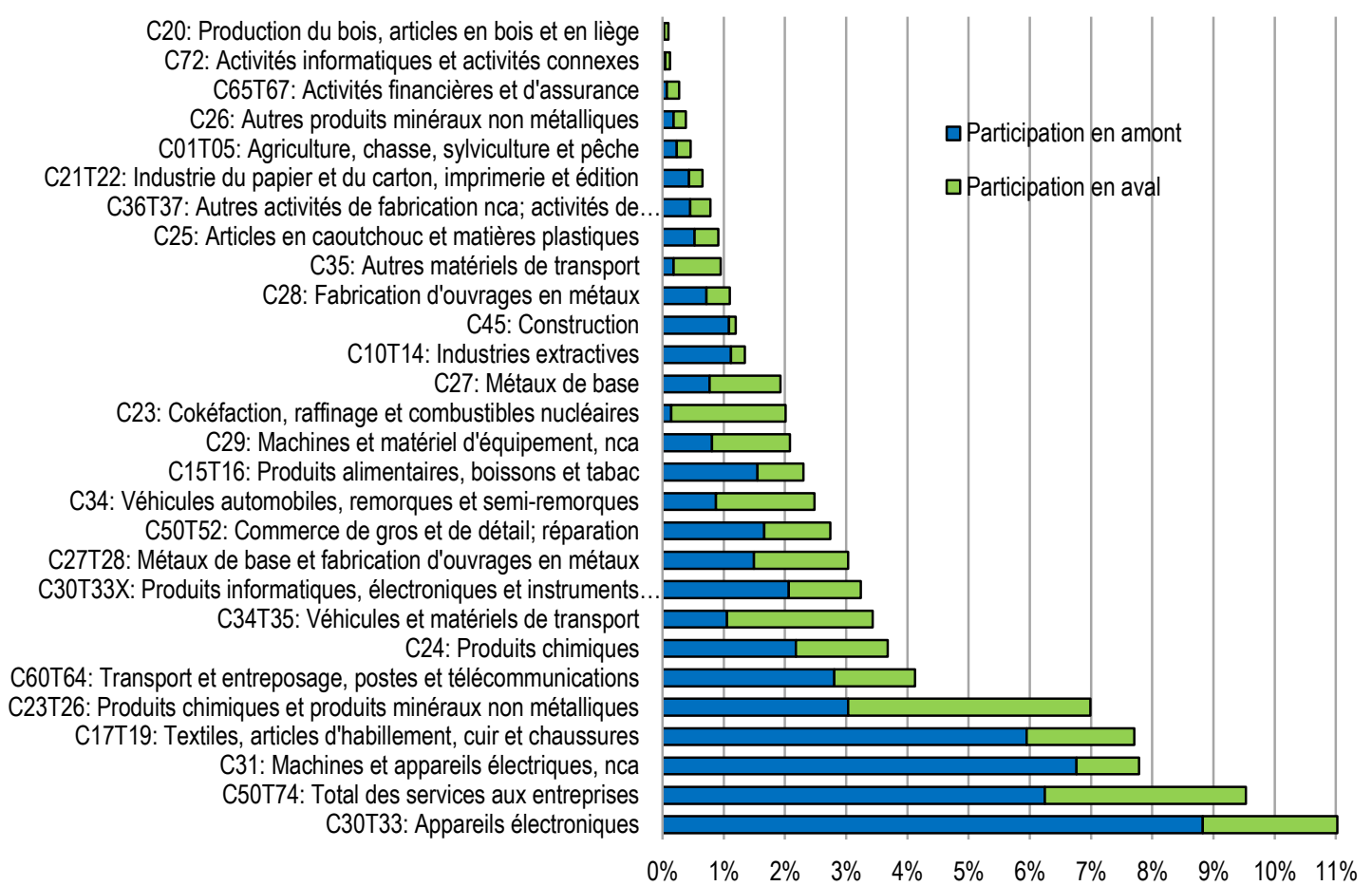

Source : Calculs des auteurs à partir des Stat. OCDE/ TiVA 2016. 


\section{Encadré 1. Commerce de services et exportations de produits manufacturés}

Les échanges de services sont l'un des segments les plus dynamiques des échanges mondiaux depuis deux décennies, et notamment les services aux entreprises. Les économies avancées occupent une position dominante dans les échanges mondiaux de services, tant à l'exportation qu'à l'importation, et disposent d'un avantage concurrentiel. Certaines économies émergentes ont néanmoins progressé rapidement dans le domaine. En particulier, I'Inde s'impose désormais comme un acteur incontournable de l'industrie informatique (OCDE, 2017a).

Les échanges de services renforcent la compétitivité du secteur manufacturier et contribuent de façon "indirecte" aux chaines de valeur mondiales. Ils peuvent réduire les coûts grâce à une meilleure gestion de la chaîne d'approvisionnement et à une meilleure ingénierie des procédés. Les échanges de services peuvent aussi contribuer au développement de produits manufacturiers de qualité pour lesquels les clients sont prêts à payer une prime. Enfin, ils incitent les prestataires locaux de services à innover et devenir plus productifs (OCDE, 2017b).

Le contenu en valeur ajoutée des services dans les exportations est relativement élevé en Tunisie, plus qu'au Maroc et plusieurs autres économies émergentes (graphique 8). Cela reflète la part élevée des exportations de services directes par rapport aux exportations totales, notamment le commerce, les transports et communications ainsi que l'hôtellerie et la restauration.

Graphique 8. Participation directe et indirecte des services dans les exportations, 2011

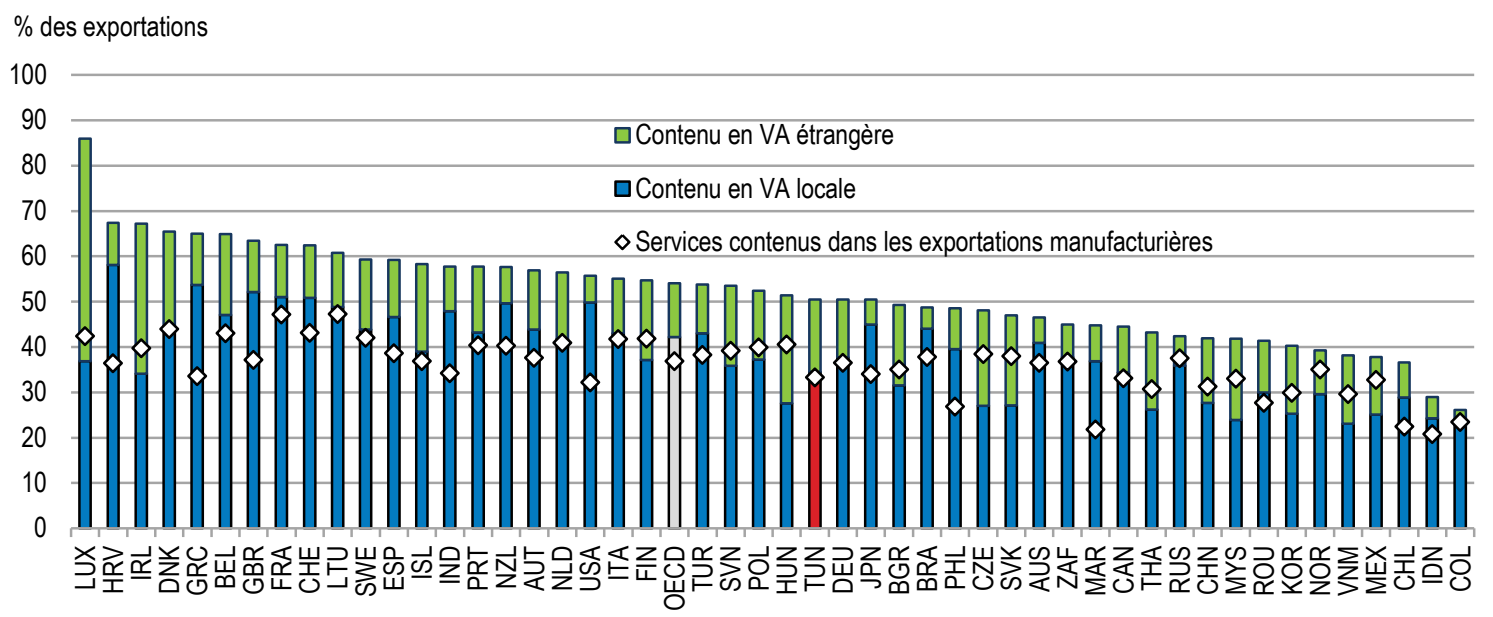

Note: La part de la valeur ajoutée locale (étrangère) du secteur des services contenue dans les exportations correspond à la proportion de valeur ajoutée générée par l'ensemble des services produits dans le pays (produits à l'étranger) dans les exportations totales. Les activités de services comprennent la construction, le commerce de gros et de détail, l'hôtellerie et la restauration, les transports et communications, les activités financières, d'immobilier et de services aux entreprises, ainsi que les services publics.

Source : TiVA/OCDE.

La valeur ajoutée des services ne représente qu'un tiers des exportations de biens manufacturés, un niveau proche de la Malaisie, l'Inde et le Mexique et supérieur à bien d'autres pays émergents, notamment le Maroc, le Chili et l'Indonésie. Certains pays font mieux dans ce domaine, notamment le Brésil, la Turquie et la République Tchèque (CITI GPS, 2018). Le manque de compétitivité dans des domaines tels que la logistique, la finance, la R\&D et les services de conception, pèse sur la contribution de la valeur ajoutée des services aux exportations de produits manufacturés.

Les restrictions à l'entrée et à l'activité dans le secteur des services constituent un frein aux exportations et importations d'ingénierie mais entravent aussi la compétitivité des entreprises manufacturières en aval (Nordas et Rouzet, 2016 ; Benz et al, 2017). L'indice de restrictivité sur les échanges de services (STRI) de l'OCDE contient des informations qualitatives sur les lois et règlements qui restreignent les échanges commerciaux de services (OCDE, 2017b). Cet indice a été construit pour 44 pays mais n'est malheureusement pas disponible pour la Tunisie.

Les données de l'OCDE concernant les restrictions aux échanges de services révèlent que peu de pays ont des 
restrictions sectorielles sur les services informatiques ; ceux-ci ont tendance à être soumis à l'environnement général des affaires. En revanche, le secteur de l'assurance est souvent soumis à une multitude de réglementations spécifiques, dont certaines interdisent explicitement ou implicitement aux étrangers d'entrer sur le marché ou augmentent considérablement les coûts d'entrée. Les restrictions à la circulation des personnes sont les plus importantes ; elles contribuent fréquemment à près de la moitié de l'indice total d'un pays. Elles sont particulièrement pénalisantes pour les services d'ingénierie qui sont intensifs en capital humain et en connaissances. Les restrictions qui entravent l'investissement direct de l'étranger peuvent également réduire les exportations (Bernard et al., 2007; Temouri et al., 2013).

L'indicateur STRI peut aussi être utilisé pour quantifier l'impact de la libéralisation dans les services tant sur le secteur lui-même que sur les autres secteurs (Nordås, 2017).

\section{Contribution du secteur offshore aux exportations, à l'emploi et au développement régional}

14. Le dynamisme des exportations et la progression de la Tunisie dans les chaines de valeurs mondiales sont étroitement liés aux investissements directs de l'étranger et aux entreprises totalement exportatrices, aussi dites entreprises offshore (encadré 2). Les entreprises offshore - exemptes de droits de douanes à l'import et l'export, bénéficiant d'un taux d'impôt réduit et surtout de procédures administratives simplifiées pour le passage des biens en douane et un meilleur accès aux services de transport contribuaient, en 2016, à 78\% des exportations de marchandises hors énergie (graphique 9). Côté importations, le régime offshore représentait 36\% du total des importations hors énergie en 2016 (graphiques 9.C \& 9.D). Au final, la participation de la Tunisie dans les CVM est principalement le fait des activités dans lesquelles la présence des entreprises offshore est forte, notamment le textile, les industries mécaniques, électriques et électroniques, la chimie et l'agro-alimentaire. Le solde commercial des entreprises offshore a progressé régulièrement pour s'établir à près de $9 \%$ du PIB en 2016. 
Graphique 9. Exportations et importations de marchandises des entreprises offshore et onshore

\section{A. Evolution des exportations par régime}
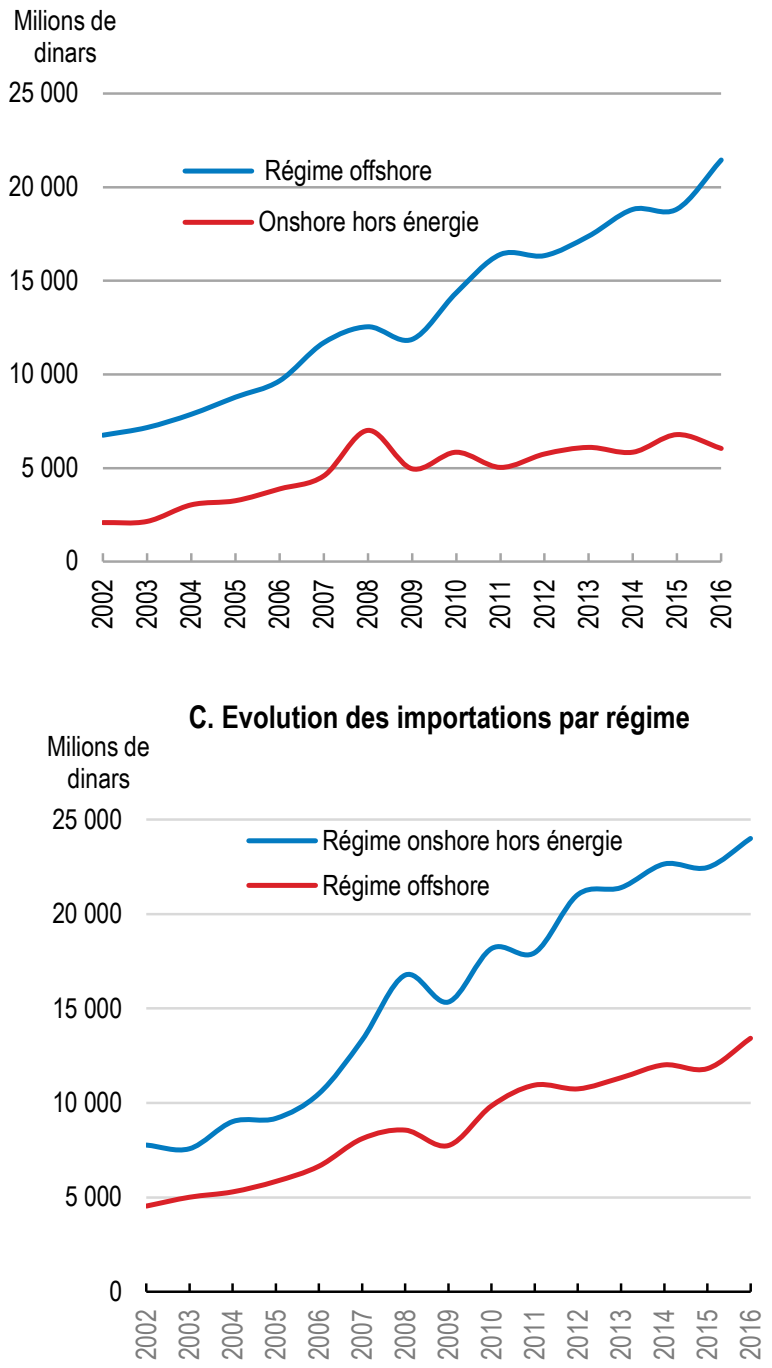

\section{B. Répartition des exportations par régime}

$\%$ des
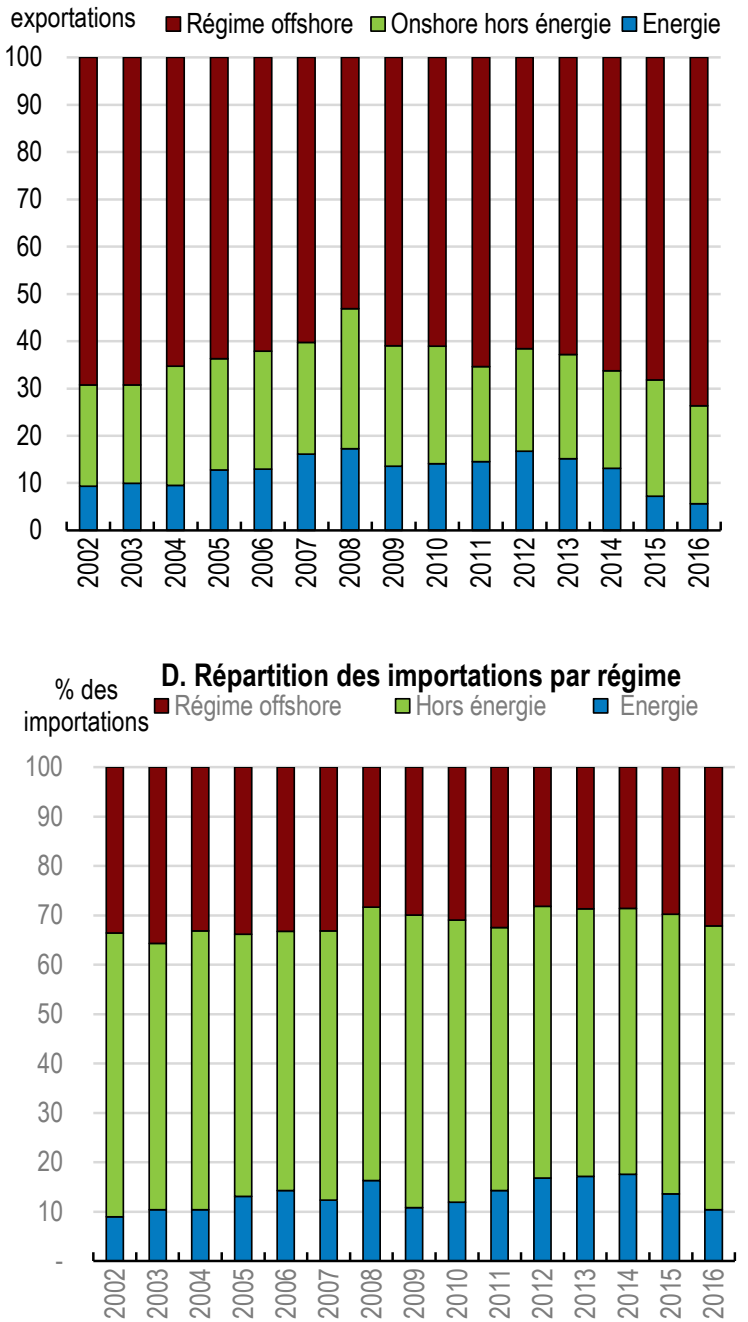

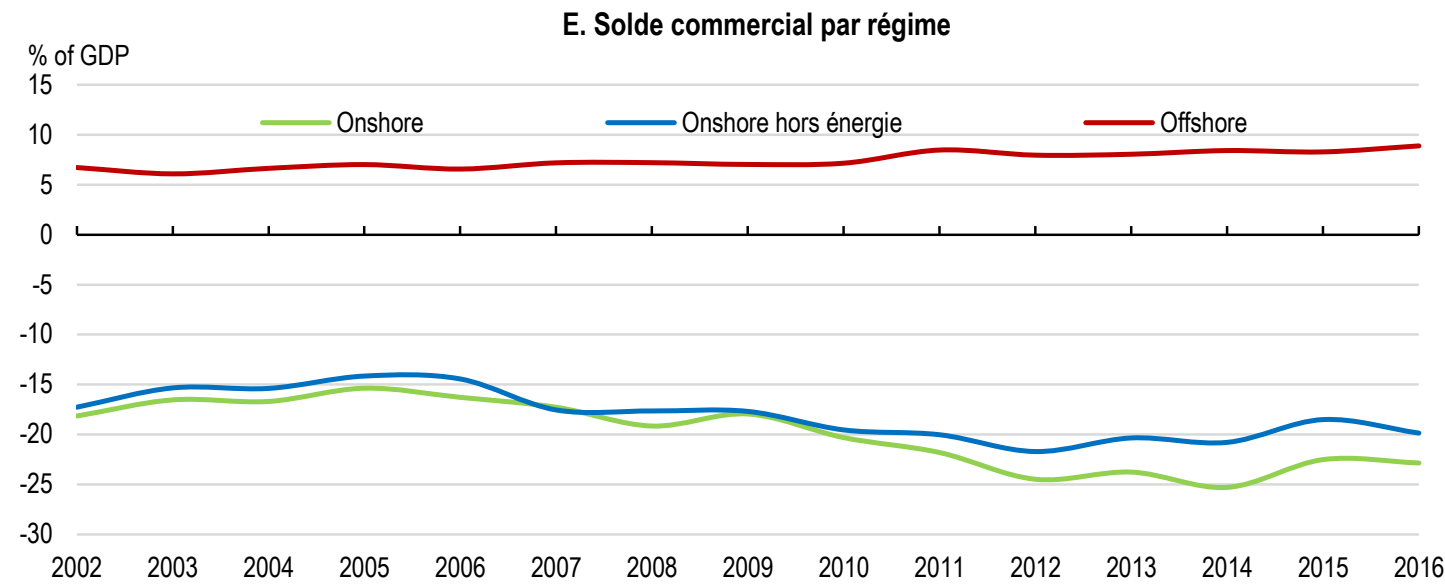

Source : INS 
Encadré 2. Entreprises totalement exportatrices et régime "offshore": définition et rôle dans l'économie

Définition du secteur offshore. Pour l'Institut National des Statistiques (INS), il s'agit des entreprises totalement exportatrices, qu'elles soient tunisiennes ou étrangères. Les sociétés totalement exportatrices sont considérées comme non résidentes lorsque leur capital est détenu par des non-résidents tunisiens ou étrangers au moyen d'une importation de devises convertibles au moins égale à $66 \%$ du capital.

Avantages concédés aux entreprises du secteur offshore. Si les entreprises offshore restent soumises aux mêmes réglementations du travail que les entreprises onshore, elles bénéficient de plusieurs avantages, notamment :

- Elles sont exonérées des droits d'importation sur les intrants incorporés dans les produits réexportés et ne traitent donc pas avec l'administration douanière si elles sont tournées exclusivement vers l'exportation.

- Elles bénéficient d'un accès privilégié aux services portuaires.

- Elles sont soumises à un taux réduit $(10 \%$ au lieu de $25 \%)$ de l'impôt sur les sociétés. Elles interagissent peu avec l'administration fiscale pendant toute la période d'exonération d'impôt. Certaines d'entre elles considèrent que cela constitue un avantage plus important que la réduction d'impôt stricto sensu.

- Lorsque le capital d'une entreprise totalement exportatrice est détenu à $66 \%$ ou plus par des nonrésidents et qu'il a été financé par importation de devises, l'entreprise est considérée comme nonrésidente "sur le plan change" et, par conséquent, n'est pas soumise à la réglementation des changes.

Depuis février 2017, les entreprises du régime offshore peuvent écouler $30 \%$ de leur chiffre d'affaires sur le marché tunisien moyennant le paiement préalable des droits de douane acquis.

\section{Participation des entreprises offshore dans l'économie tunisienne}

Le nombre des entreprises offshore a été multiplié par plus de 14 entre 1996 et 2016, alors que celui des entreprises du secteur onshore était multiplié par 2 selon le Répertoire National des Entreprises (RNE). Leur part dans l'emploi salarié formel du secteur privé a presque doublé depuis 1996 pour s'établir à 34\% (graphique 10) -- la part du secteur offshore s'élevait ainsi à $19.3 \%$ de l'emploi total. Les entreprises offshore sont, en moyenne, plus grandes que leurs homologues onshore. Les grandes et moyennes entreprises (c.a.d. avec un effectif au moins égal à 50) représentent près de $95 \%$ du total des entreprises offshore contre $67.4 \%$ dans le régime onshore selon l'Agence de Promotion de l'Industrie et de l'Innovation. Dans le secteur industriel, les entreprises offshore représentent $44 \%$ des entreprises employant au moins 10 salariés et $64 \%$ des emplois.

\section{Graphique 10. Entreprises et emplois dans le secteur offshore}

A. Nombre des entreprises privées bénéficiant du régime offshore

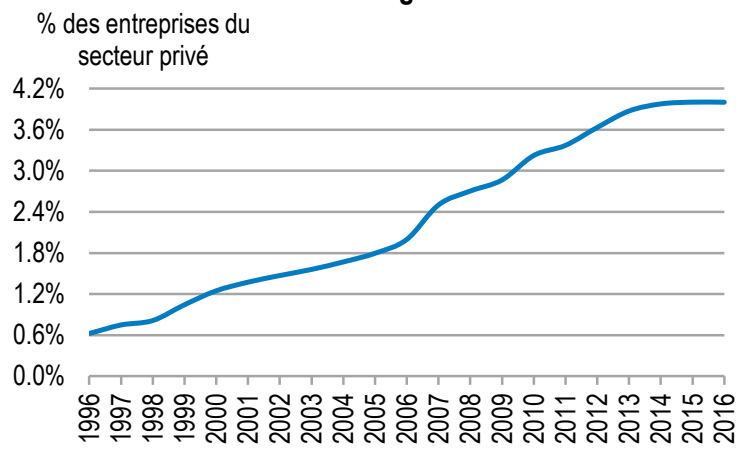

B. Emploi salarié formel dans les entreprises offshore

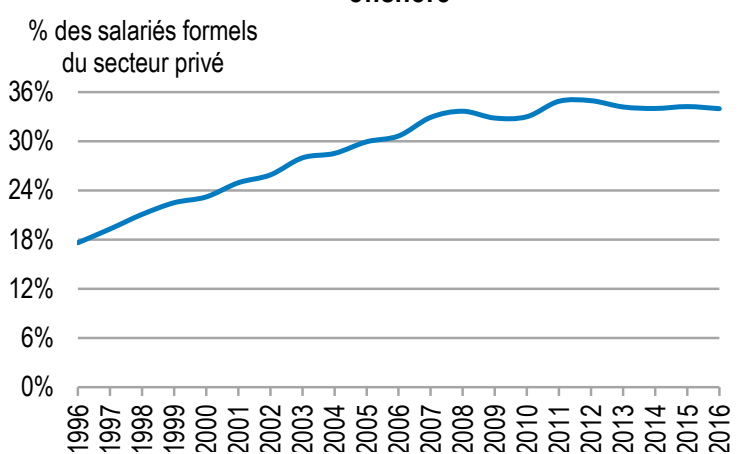

Source: INS. 


\title{
Entreprises offshore et développement régional
}

Les entreprises offshore sont fortement concentrées dans les régions et gouvernorats côtiers, profitant de la proximité des ports commerciaux par lesquels transitent l'essentiel du commerce de marchandises en Tunisie. Ainsi, selon les données du RNE, 72\% des entreprises offshore étaient installées dans les régions du Nord-Est en 2012 et $24 \%$ dans celles du Centre-Est (graphique 11). La moitié des entreprises offshore étaient installées dans deux gouvernorats - Tunis et Ariana --, l'autre moitié se répartissant entre les 22 gouvernorats restants. Les enquêtes auprès des entreprises suggèrent que les difficultés d'accès aux ports mais aussi la faible disponibilité des services administratifs dans les régions intérieures constituent des contraintes à leur installation dans les régions de l'intérieur. II faut aussi noter que les données sur la localisation concernent le siège social et ne reflètent pas entièrement la localisation de l'emploi, créant ainsi un biais dans l'analyse.

\section{Graphique 11. Répartition des entreprises offshore par région, 2016}

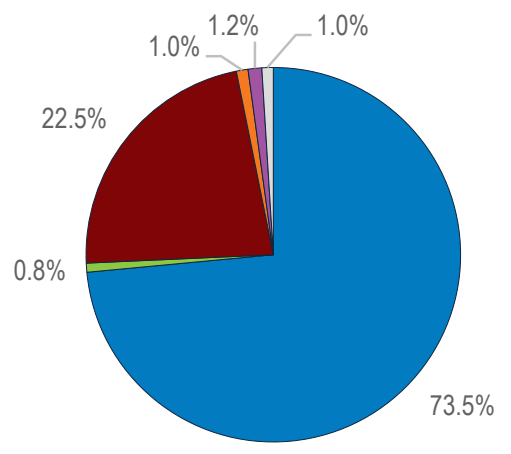

\author{
a Nord-Est \\ $\square$ Nord -Ouest \\ - Centre-Est \\ $\square$ Centre-Ouest \\ $\square$ Sud-Est \\ $\square$ Sud-Ouest
}

Source : Répertoire national des entreprises de l'INS

\section{Graphique 12. Intensité de présence des entreprises offshore par gouvernorat}
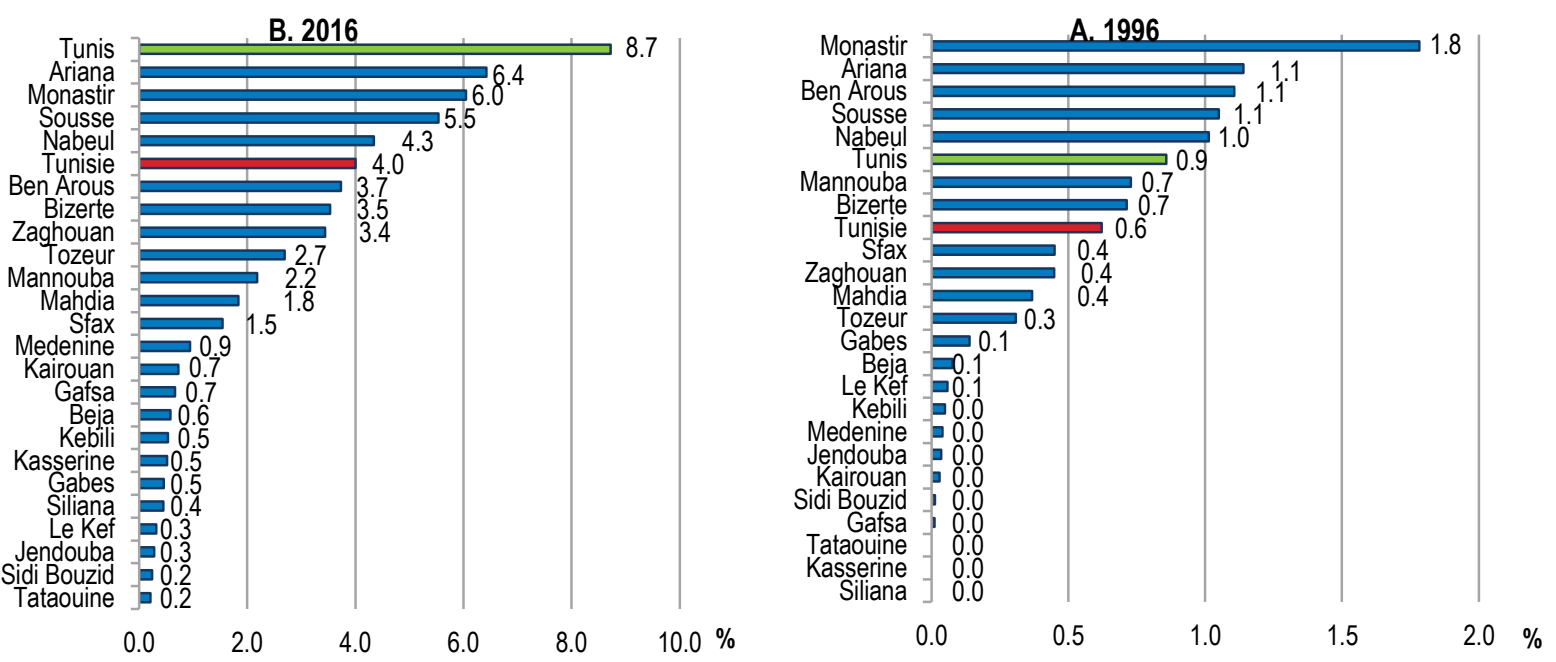

Note: L'intensité de la présence des entreprises offshore est définie comme la part des entreprises offshore dans le total des entreprises.

Source : Calcul des auteurs à partir du Répertoire nationale des entreprises de l'INS.

Un mouvement de rééquilibrage entre les régions semble néanmoins se dessiner, avec une augmentation de la part des entreprises offshore dans les gouvernorats de l'intérieur entre 1996 et 2016 (graphique 12). Certaines entreprises offshore se sont installées plus récemment dans les régions de l'intérieur où la concurrence pour le personnel qualifié est moins rude, une dynamique confirmée par les données de l'APII sur les entreprises de plus de 10 salariés. 
15. Les entreprises qui n'opèrent pas dans le régime offshore souffrent de la multiplicité des droits de douane, laquelle s'accompagne souvent de contrôles sur la nature des biens importés et de tracasseries administratives. Cela génère des coûts et nuit à la compétitivité à l'exportation des entreprises onshore. En effet, l'efficacité des services logistiques autour des infrastructures portuaires est faible, ce qui pèse sur la qualité et la disponibilité des services de manutention et de transport de marchandises (graphique 13.A). En outre, la Tunisie s'est laissée distancer par ses concurrents directs sur des indicateurs-clé comme le temps d'attente à la frontière ou l'efficacité des services douaniers. Les données de l'OCDE sur la facilitation des échanges suggèrent aussi que les procédures aux frontières qui s'appliquent aux entreprises onshore se sont détériorées (graphique 13.B). En outre, les restrictions à la concurrence, et en particulier le système des licences et autorisations (OCDE, 2018), incitent peu les entreprises en place du régime onshore à se diversifier et à améliorer leur compétitivité. De fait, plus de $60 \%$ des exportations des entreprises onshore sont des exportations de produits agricoles, énergétiques, miniers et phosphatés, qui se caractérisent par une chaine de production relativement courte.

\section{Graphique 13. Performance en logistique de transport et facilité à passer les biens en douane}

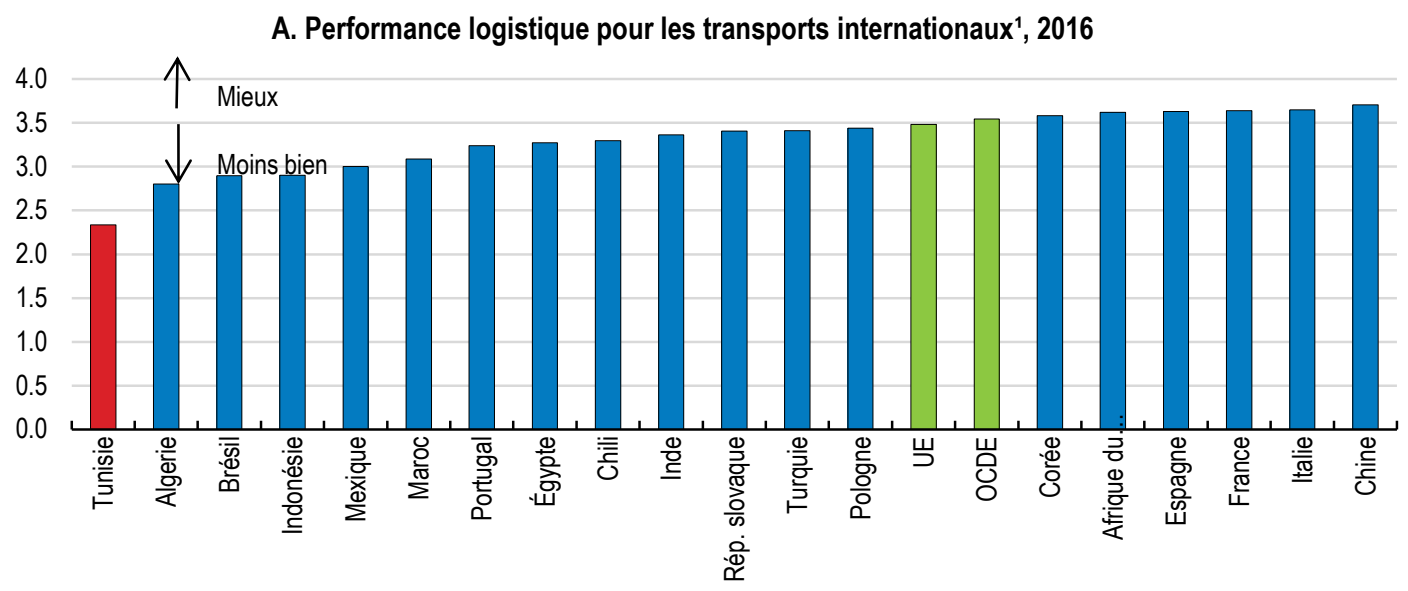

B. Indicateur de l'OCDE sur la facilitation des échanges ${ }^{2}, 2017$

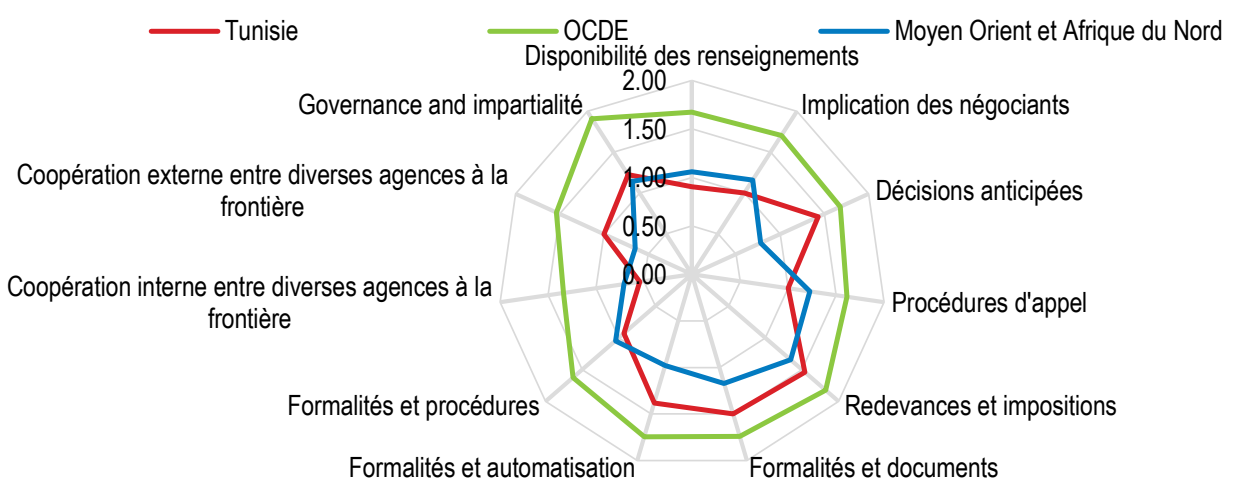

1. La note globale de l'indice de performance de la logistique reflète les perceptions relatives à la logistique d'un pays basées sur l'efficacité des processus de dédouanement, la qualité des infrastructures commerciales et des infrastructures de transports connexes, la facilité de l'organisation des expéditions à des prix concurrentiels, la qualité des services d'infrastructure, la capacité de suivi et de traçabilité des consignations et la fréquence avec laquelle les expéditions arrivent au destinataire dans les délais prévus. L'indice va de 1 à 5 et la note la plus élevée représente la meilleure performance.

2. Les indicateurs de facilitation des échanges mesurent l'incidence économique et commerciale relative des mesures de facilitation actuellement négociées, dans le cadre de l'Organisation mondiale du commerce (OMC), sur les flux et les coûts d'échanges. L'indicateur varie de 0 à 2; plus il est élevé, meilleure est la performance.

Source : World Bank Logistics Performance Index database ; et OECD Trade facilitation indicators database. 
16. Outre leur faible participation à l'exportation et aux chaines de valeur mondiales, les entreprises du secteur onshore entretiennent peu de liens économiques avec les entreprises du secteur offshore. Lorsqu'elles écoulent leur production sur le marché tunisien, les entreprises totalement exportatrices doivent s'acquitter des impôts et droits de douanes, donc entrer en relation avec l'administration. Les entreprises totalement exportatrices peuvent s'approvisionner sur le marché local et, dans ce cas, sont exonérées de la taxe sur la valeur ajoutée. Dans la pratique, elles le font peu. De la même façon, une entreprise onshore qui vend des produits à une entreprise offshore devrait pouvoir se voir rembourser la TVA payée sur ses consommations intermédiaires. Dans la pratique, les remboursements sont effectués une fois par an, générant des problèmes de trésorerie pour certaines entreprises, en particulier les petites. Certaines entreprises notent que la demande du crédit de TVA est aussi souvent suivie de contrôles comptables et fiscaux qui découragent la sous-traitance par les entreprises onshore. La fiscalité, les procédures administratives et douanières constituent une barrière au développement des relations de soustraitance entre les deux secteurs et plus généralement aux effets d'entrainement du secteur offshore sur l'économie tunisienne. Au final, les entreprises des deux régimes échangent peu et les transferts de technologie, souvent associés à l'exposition au commerce et aux investissements étrangers, sont inhibés (Dhaoui et Samoud, 2016).

\section{Secteur offshore et investissements directs}

17. L'investissement direct étranger et le secteur offshore sont fortement liés mais ne sont pas synonymes. Les entreprises offshore représentaient $80 \%$ des entreprises étrangères installées en Tunisie en 2016 (RNE 2017/INS); et, selon les données de l'APII portant sur les entreprises employant au moins 10 salariés, 6 entreprises offshore sur 10 sont à participation étrangère (APII-2018). La forte présence des entreprises étrangères est allée de pair avec des investissements directs étrangers élevé au cours des dernières décennies, confirmant l'attractivité et la compétitivité de la Tunisie. En effet, la loi de 1972 a institué des avantages à l'investissement étranger et le secteur exportateur au travers d'un régime spécial qui vise à encourager les entreprises à investir, à créer des emplois et à exporter, grâce à la création de sociétés totalement exportatrices. Ces avantages ont été renforcés par la promulgation du code d'incitations à l'investissement en 1993. Au final, le stock d'investissement direct étranger, en pourcentage du PIB, est très élevé par rapport à la plupart des autres pays et les flux d'entrée ont plutôt bien résisté ces dernières années (graphique 14). La mise en œuvre de la nouvelle loi sur l'investissement à partir de 2017 se traduira par une réduction des restrictions à l'investissement direct étranger (OCDE, 2018), ouvrant la voie à une nouvelle hausse des IDEs.

18. L'analyse de la répartition sectorielle des flux d'investissement direct de l'étranger corrobore les faits observés sur la nature des exportations. Elle met en évidence une orientation plus marquée vers les industries électriques et électroniques, suivies par le tourisme, les industries mécaniques, les télécommunications, l'agro-alimentaire et l'industrie pharmaceutique. Parmi les industries manufacturières, le secteur textile, peu intense en technologies et en capital humain, a perdu du terrain au profit des secteurs plus intenses en capital humain et en technologie. Ainsi, les IDE se concentrent davantage dans des secteurs à fort potentiel en matière d'intégration dans les CVM et de positionnement à l'international (graphique 15). 
Graphique 14. Les flux et stocks d'investissements directs étrangers sont plutôt élevés

\section{A. Investissements directs étrangers, entrées nettes'}
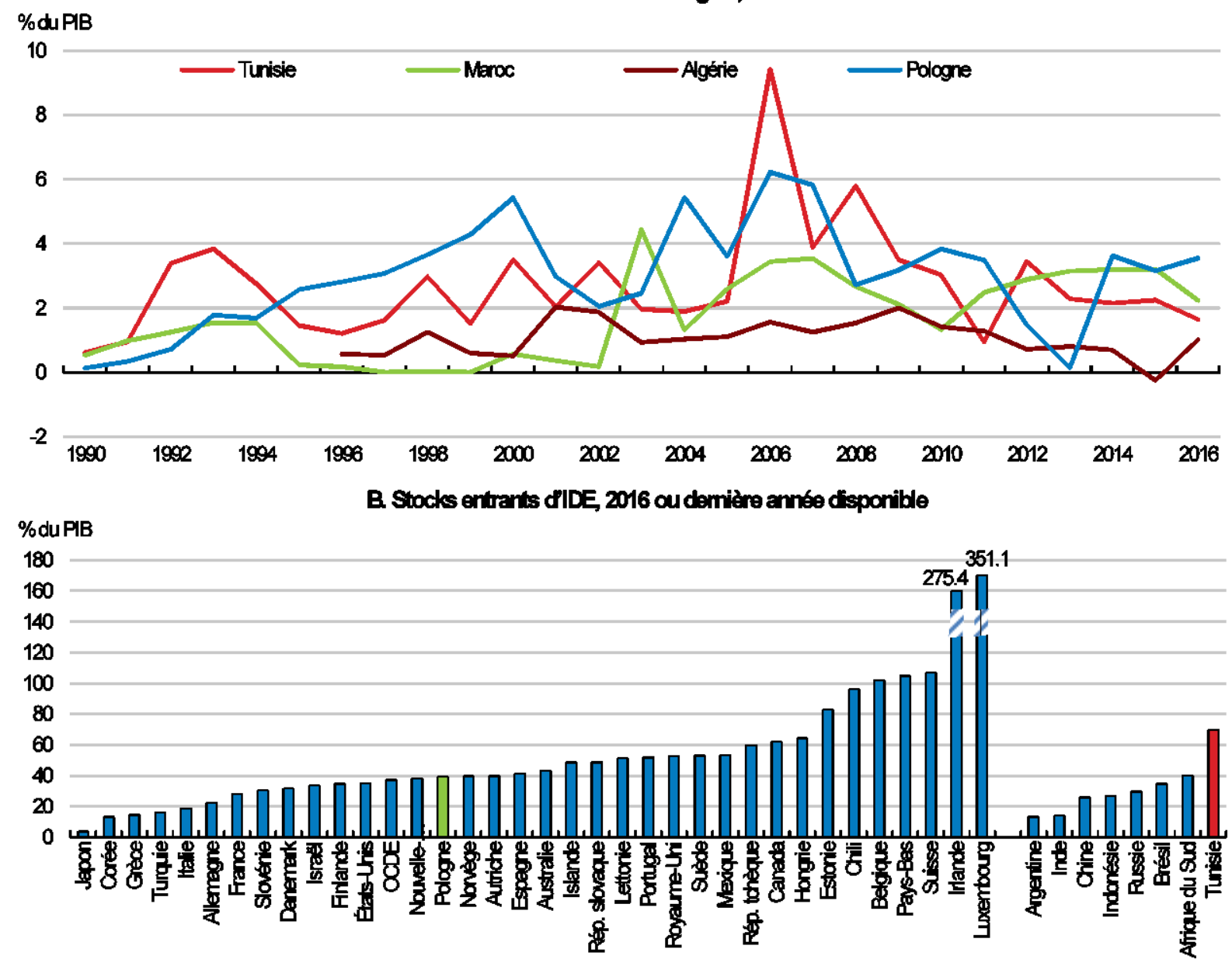

Source: Banque mondiale, Indicateurs de développement mondial (WDI) ; OCDE base de données des Principaux agrégats d'IDE; Base de données des Perspectives de l'économie mondiale du FMl; et base de données de la balance des paiements du FMI. 


\section{Graphique 15. Évolution de la structure des investissements directs de l'étranger}

A. Répartition des IDE par secteur d'activité ${ }^{1}$ en 1996 et 2017

1996

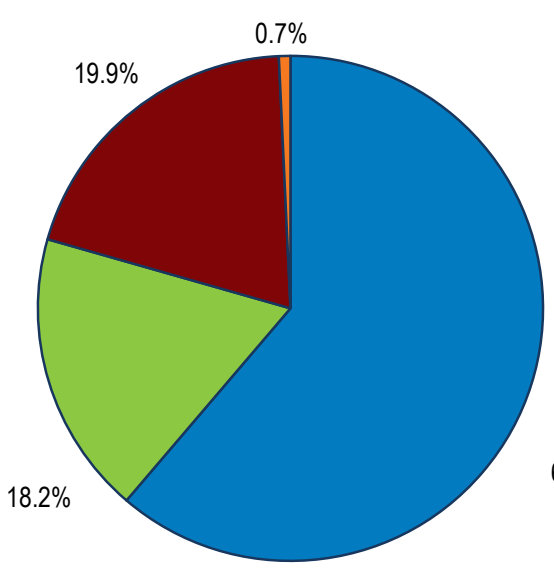

\section{aEnergie}

口Ind. manufacturières

- Services

$\square$ Agriculture

$61.2 \%$

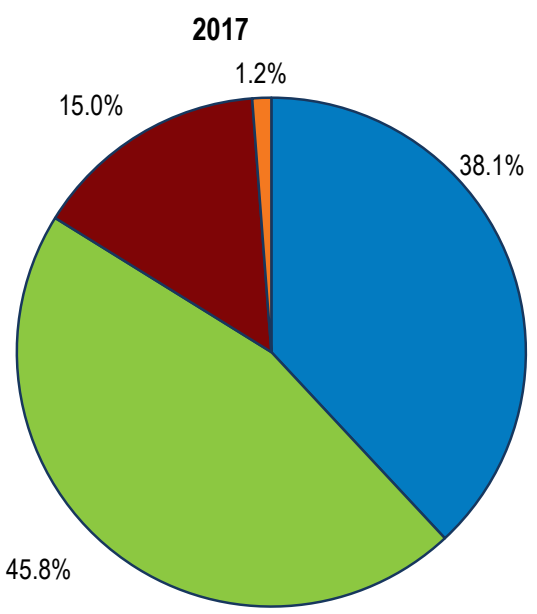

\section{B. Répartition des IDE manufacturiers en 1995 et 2017}
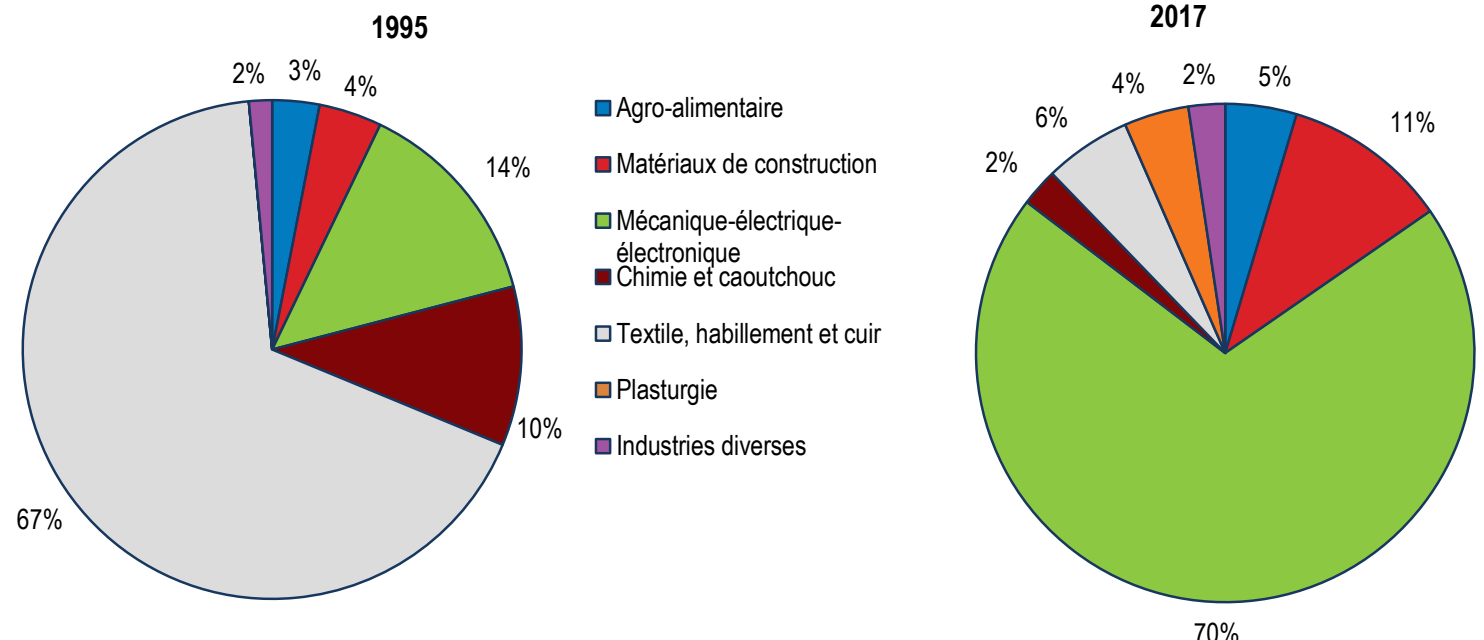

1. Calcul des auteurs à partir des données de la BCT pour l'année 1996 et de la FIPA pour l'année 2017.

Source: Calcul des auteurs à partir des données de la BCT et de la FIPA. 


\section{BIBLIOGRAPHIE}

Benz, S. A. Khanna et H.K. Nordås, (2017), Services and performance in the Indian economy: Analysis and policy options, OECD Trade Policy Paper No. 196.

Bernard, A.B., J. B Jensen, S.J. Redding and P.K. Schott (2007), Firms in international trade, Journal of Economic Perspectives, 21, 105-130.

CiTi GPS (2018), Securing India's growth over the next decade: twin pillars of investment and productivity", Citi GPS: Global Perspectives \& Solutions, February 2018.

Dhaoui. S et Sammoud. I (2016): «Investissement direct étranger et transfert de technologie : cas des industries manufacturières tunisiennes »; ITCEQ (2016)/ Notes et Analyses de l'ITCEQ n¹3/ Avril 2016

Égert, B. and P. Gal (2017), The Quantification of Structural Reforms in OECD Countries: A New Framework, OECD Economics Department Working Papers, No. 1354, OECD Publishing, Paris. DOI: http://dx.doi.org/10.1787/2d887027-en

Fajgelbaum, P. et A. Kandhelwal (2016), Measuring the unequal gains from trade, QJE, Vol. 131.

Hausmann R, C. A. Hidalgo , B. Klinger B, et A. L. Barabási (2007), The product space conditions the development of nations. Science, 2007 Jul 27;317(5837):482-7.

Hausmann R., C. A. Hidalgo., S. Bustos, M. Coscia, A. Simoes et M. Yildirim (2011): ,Atlas of Economic Complexity: Mapping Paths to Prosperity, Massachusetts Institute of Technology and Center for International Development, Harvard University.

Hausmann R. et D. Rodrik ( 2003), Economic development as self-discovery, Journal of Development Economics, vol. 72, issue 2, 603-633

Nordås H. K. (2017), Service trade restrictiveness index, methodology and application: the Indian context, OECD [s'assurer auprès de l'auteur de la citation, dans quel livre?](dans le papier s'est ecrit OCDE)

Nordås, H.K. and D. Rouzet (2016), The impact of services trade restrictiveness on trade flows, World Economy, doi: 10.1111/twec.12424

OCDE (2017a), Perspectives économiques de l'OCDE, juin 2017, $\mathrm{N}^{\circ} 101$.

OCDE (2017b), Services Trade Policies and the Global Economy, OECD Publishing, Paris http://dx.doi.org/10.1787/9789264275232-en

OCDE (2017d), "Comment rendre le commerce mondial bénéfique pour tous", Perspectives économiques de l'OCDE, Juin 2017, chapitre 2.

OCDE (2018), Étude économique de l'OCDE sur la Tunisie, Paris.

Temouri, Y., A. Vogel and J. Wagner (2013), Self-selection into export markets by business services firms - Evidence from France, Germany and the United Kingdom, Structural Change and Economic Dynamics, 25, 146-158. 


\section{ANNEXES}

Annexe 1. Complexité et sophistication des exportations-espace produit entre 1995 et 2016

Tunisie 1995

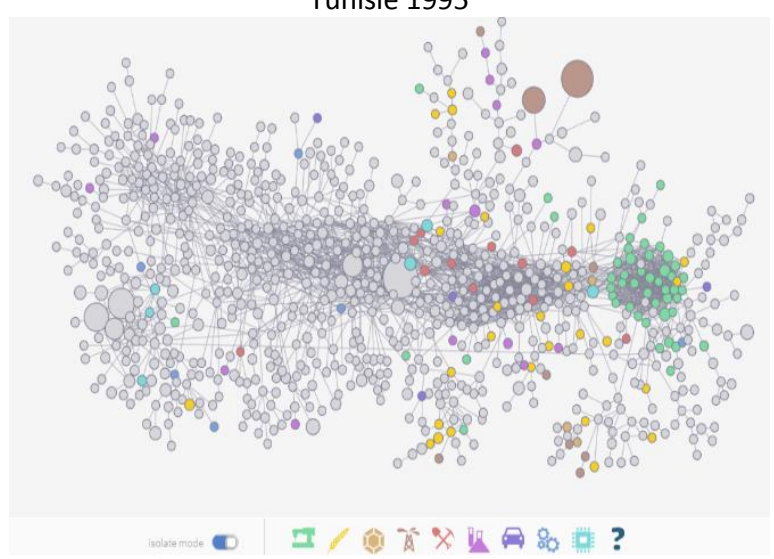

Maroc 1995

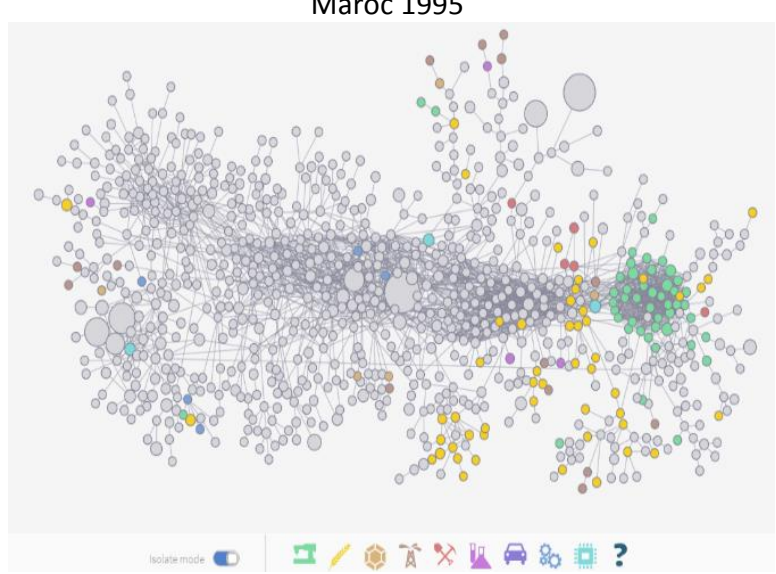

Turquie 1995

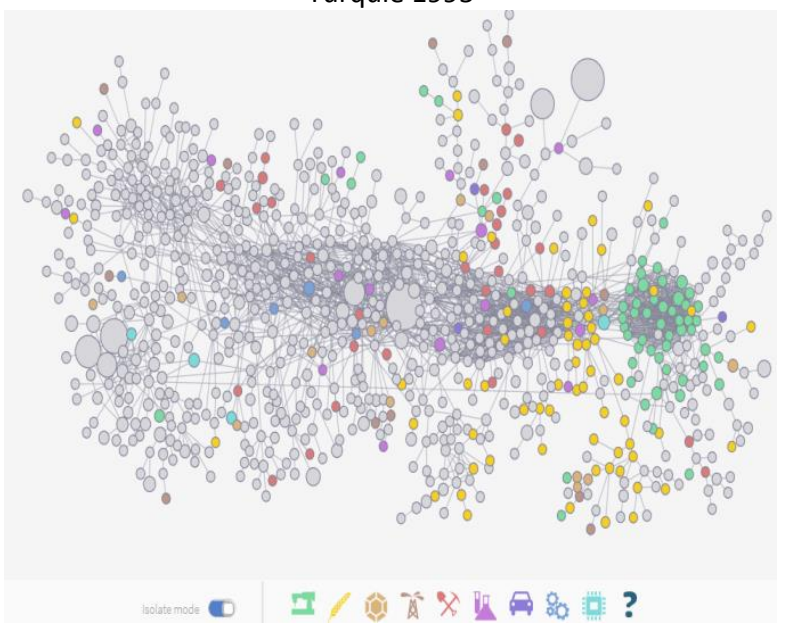

Tunisie 2016

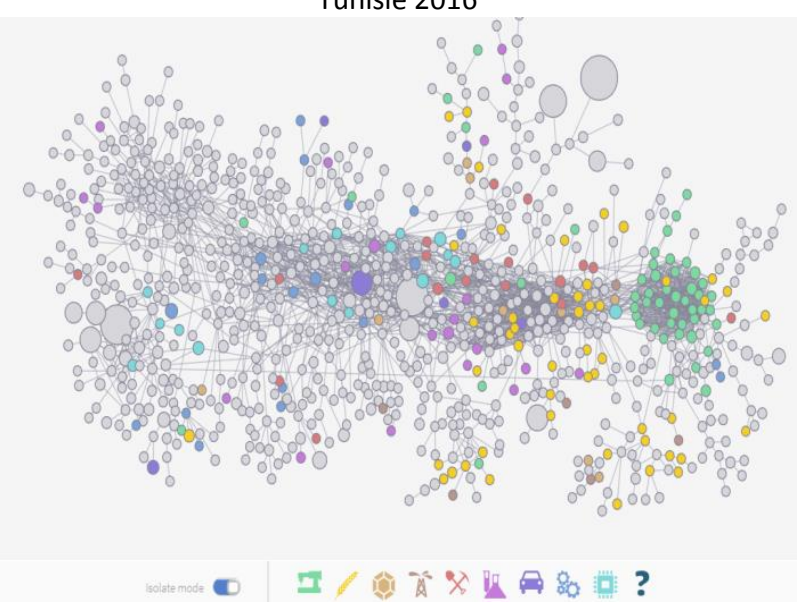

Maroc 2016

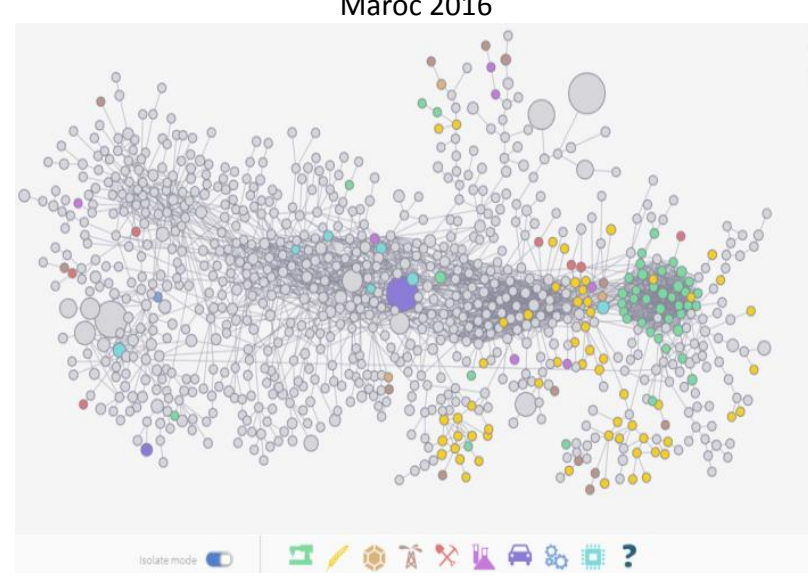

Turquie 2016

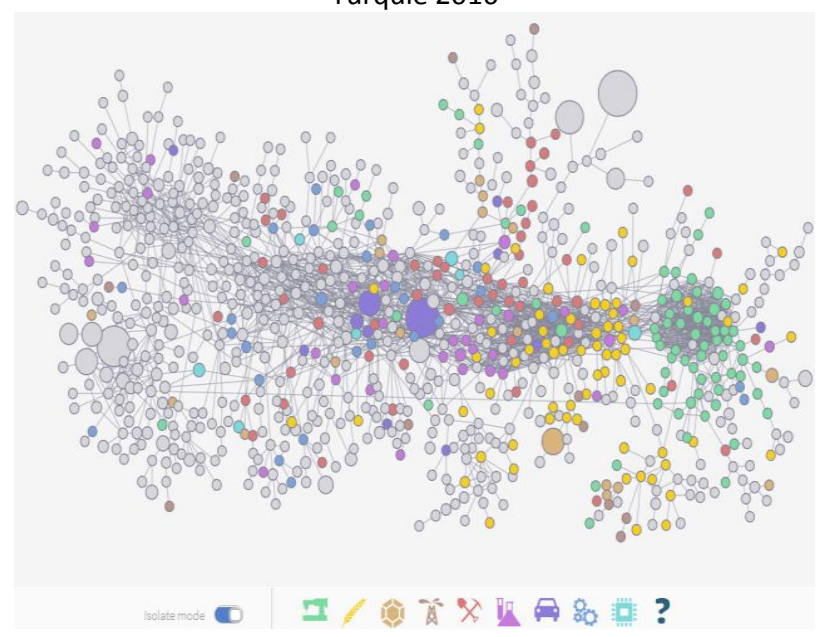




\section{$\mathrm{ECO} / \mathrm{WKP}(2018) 26$}

Egypte 1995

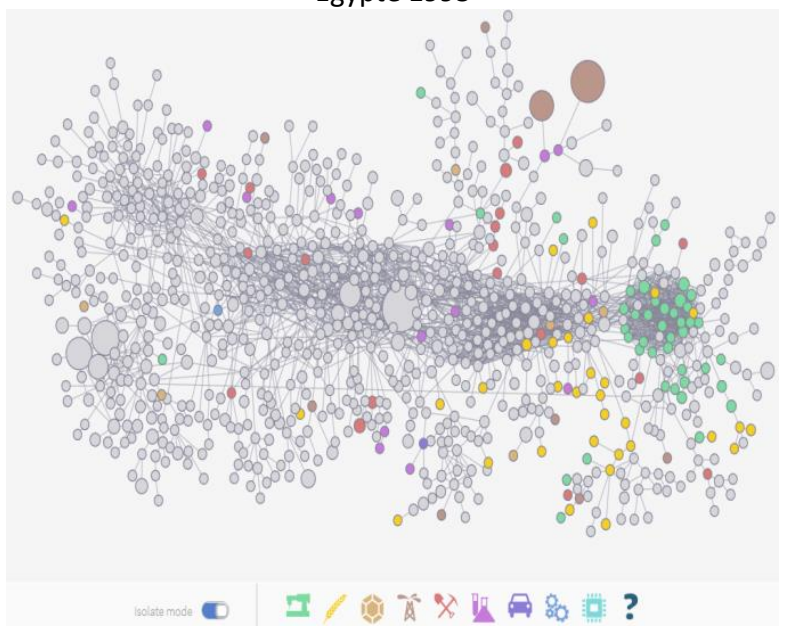

Rep. Tchèque 1995

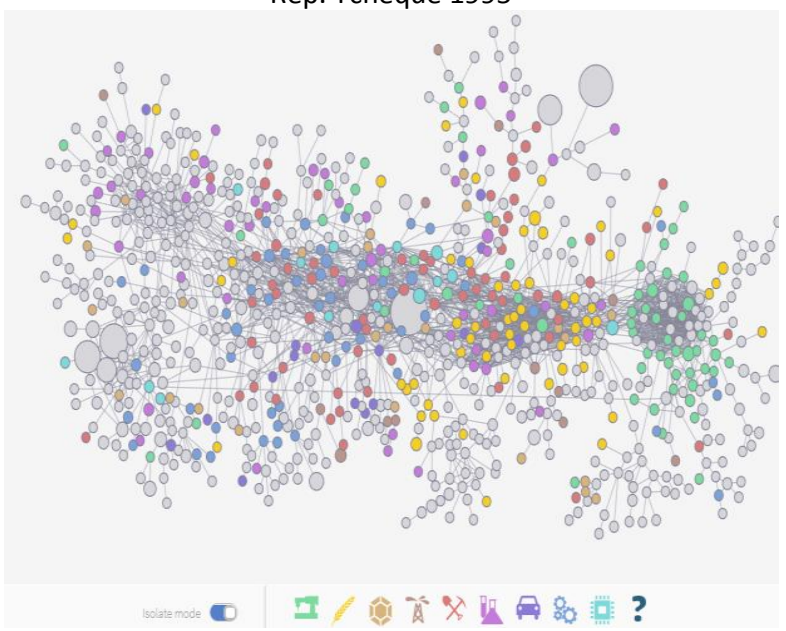

Egypte 2016

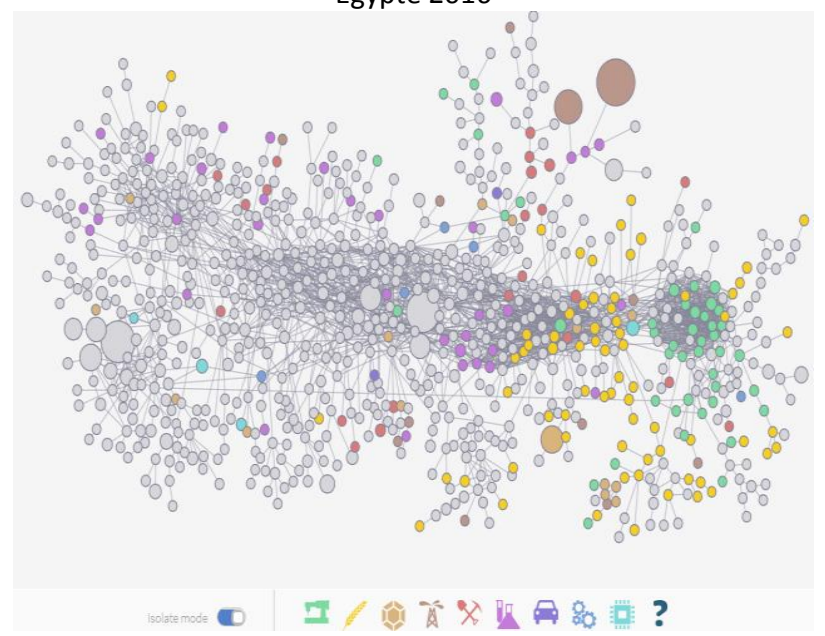

Rep. Tchèque 2016

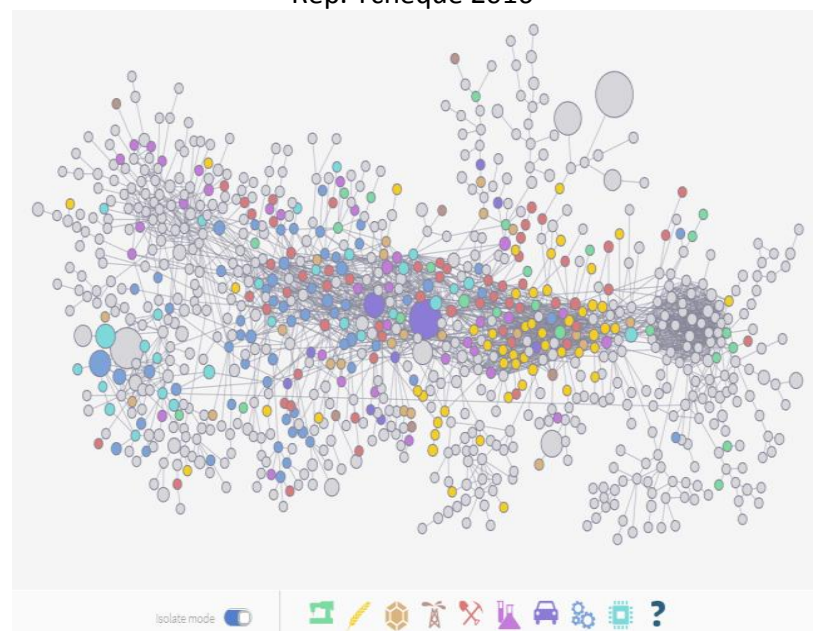

Source: The Observatory of Economic Complexity (OEC).

Note: L'espace produit est une visualisation qui représente un réseau de produits susceptibles d'être co-exportés et pouvant servir à prédire l'évolution de la structure d'exportation d'un pays. L'organisation des produits dans cet espace est déterminée par la similarité ou la dissemblance de leurs exigences en matière de connaissances. En utilisant des données sur les exportations d'un pays, l'OEC génère un espace produit qui visualise ce que ce pays est en mesure de fabriquer, les produits qui sont à proximité et que le pays pourrait commencer à fabriquer, et peut donc aider à déterminer les voies de l'expansion industrielle. La concentration au milieu de l'espace produit signifie que les produits exportés avec un avantage comparatif révélé sont plus complexes et plus sophistiqués.

\section{Complexité économique}

La complexité d'une économie reflète la variété des connaissances utilisées. Elle peut se mesurer par la composition des biens que les pays sont capables de produire. L'indice de complexité économique permet de classer les économies en fonction de la complexité de leur panier d'exportations. Plus la complexité est élevée, plus le rang de classement est faible, plus ses perspectives de croissance sont élevées.

\section{Sophistication des exportations}

Selon Hausmann et Rodrik (2003) et Hausmann et al. (2007), la structure du panier des produits exportés par un pays affecte la création de nouvelles lignes d'exportation affectant à leur tour le son potentiel de croissance. Toutes choses égales par ailleurs, les pays qui se spécialisent dans les produits exportés par les pays ayant des niveaux de revenu par habitant élevés sont susceptibles d'améliorer leur croissance plus vite que ceux produisant les autres marchandises. Le concept de la sophistication des produits utilisé dans la nouvelle théorie du commerce international revient à générer pour chaque produit un couple revenu/productivité. Ce couple donne une idée sur le niveau de productivité atteint par le produit ainsi que sa valorisation monétaire basée sur la moyenne des recettes des exportations. 
ECO/WKP(2018)26

Légende

\begin{tabular}{|l|l|l|l|}
\hline & Textile-habillement-cuir-chaussures & Produits chimiques, Plastiques et caoutchouc \\
\hline & $\begin{array}{l}\text { Produits animaux et végétaux, produits d'origine } \\
\text { animale, produits alimentaires et bois }\end{array}$ & Véhicules et matériels de transport \\
\hline & Pierre et verre & Machines \\
\hline & Produits minéraux & Produits électroniques \\
\hline
\end{tabular}




\section{Annexe 2.}

Figure A. Participation du secteur manufacturier dans les CVM

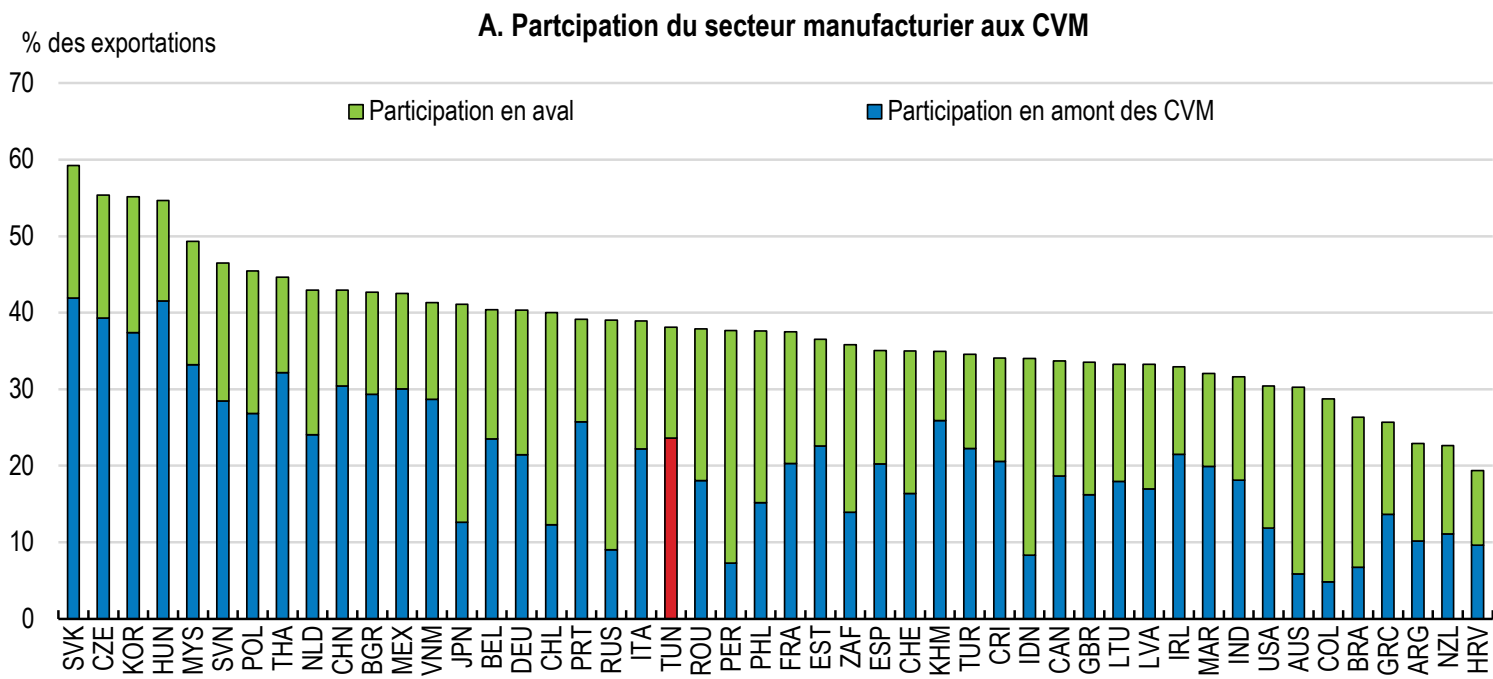

B. Changement dans la participation du secteur manufacturier dans les CVM entre 2005 et 2011

Points de pourcentage

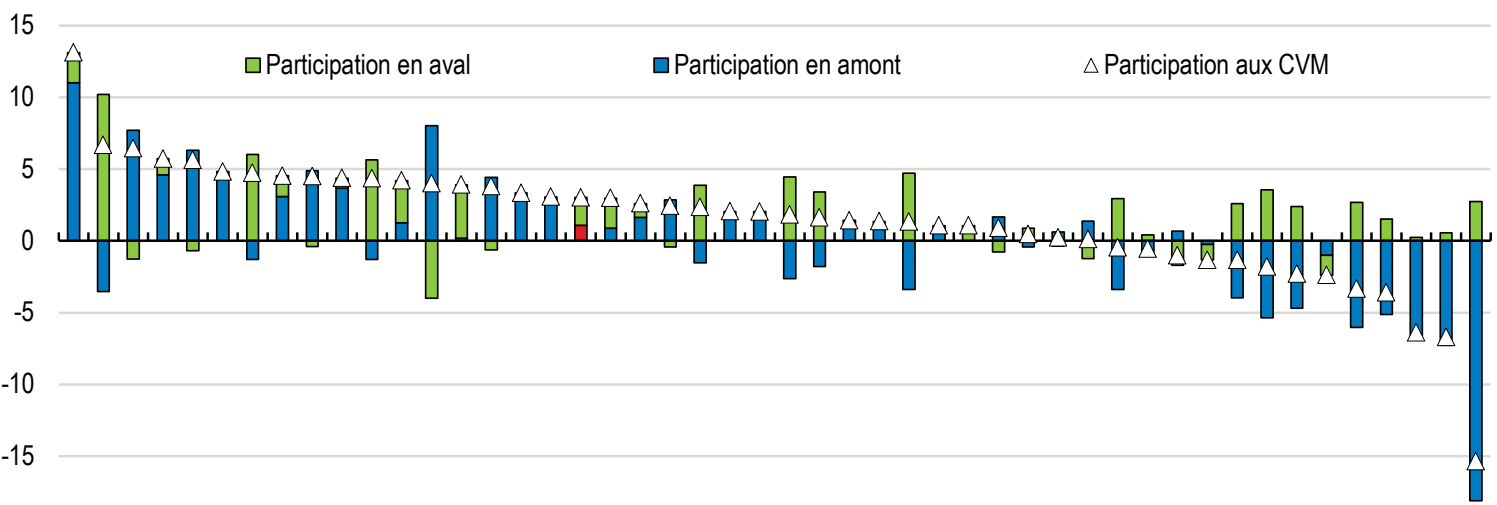

$-20$

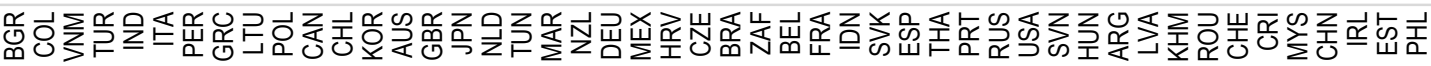



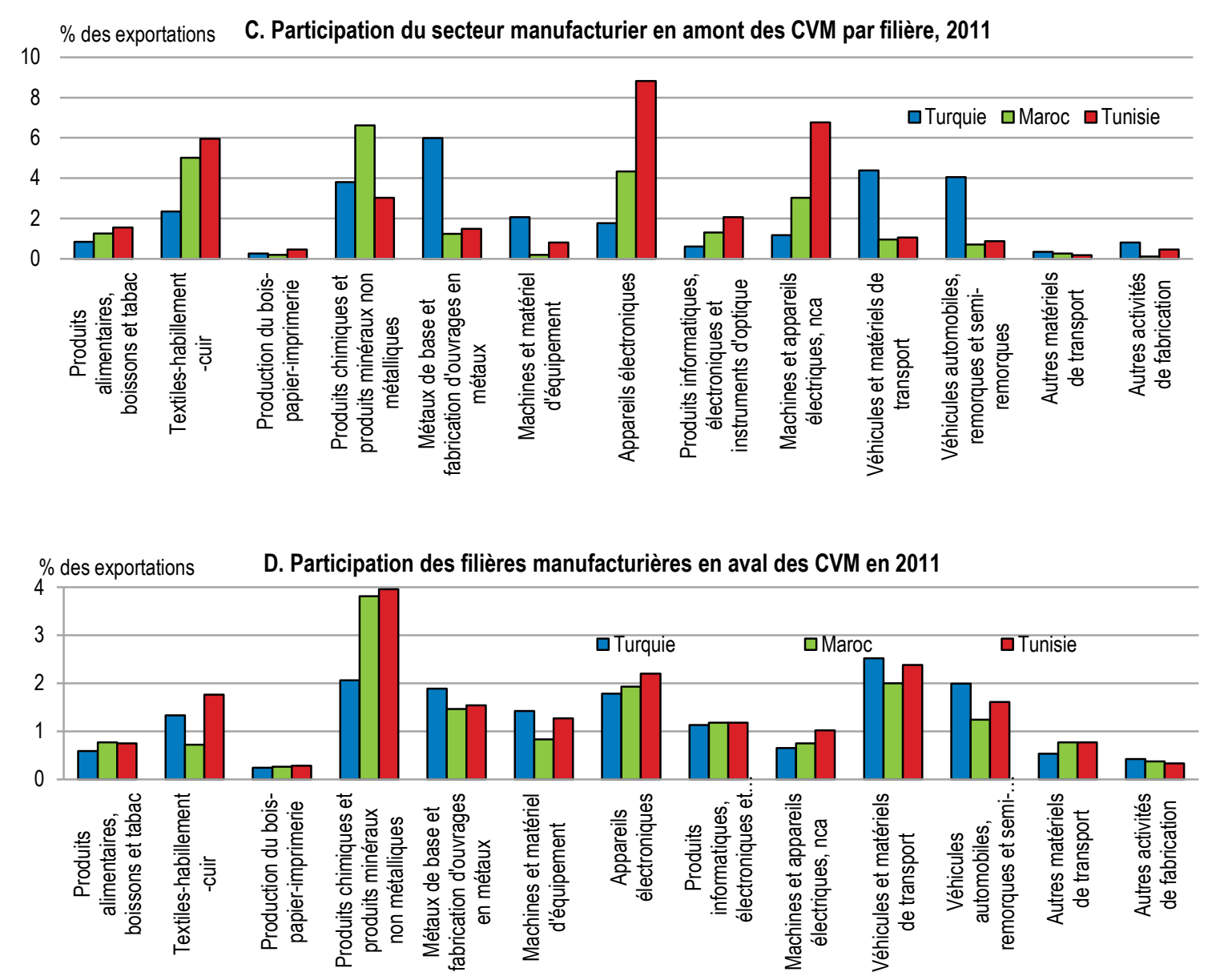

Source: TiVA database/ OCDE-2016 ; et calculs des auteurs à partir des données de la base TIVA/ OCDE-2016. 
Figure B. Participation du secteur des services dans les CVM

A. Participation du secteur des services aux CVM en 2011

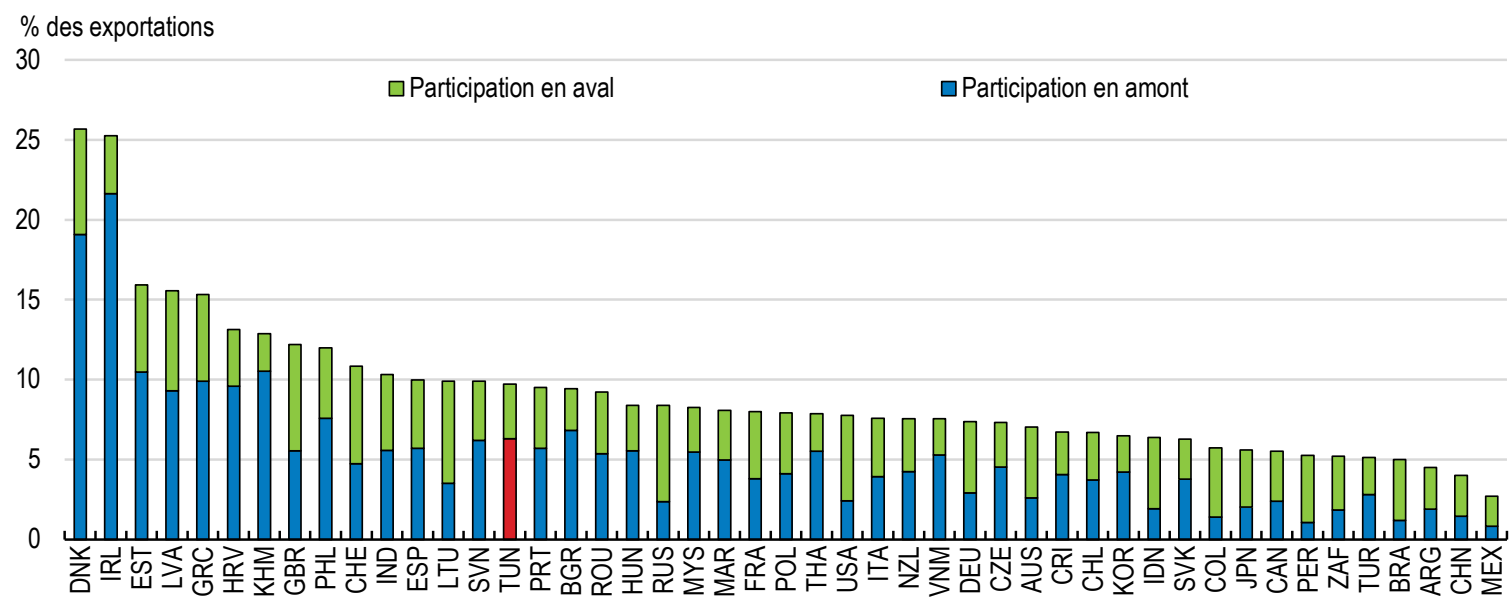

B. Changement dans la participation du secteur des services aux CVM entre 2011 et 2005

Points de pourcentage

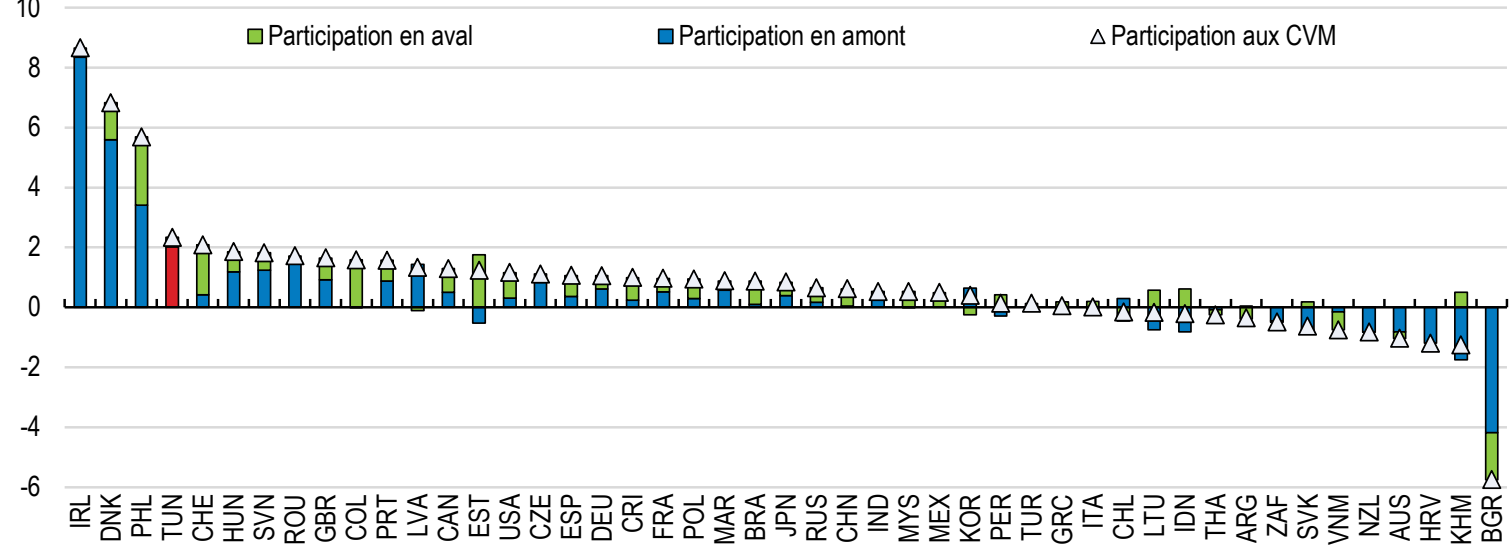


ECO/WKP(2018)26

\section{Participation des filières de services en amont des CVM en 2011}
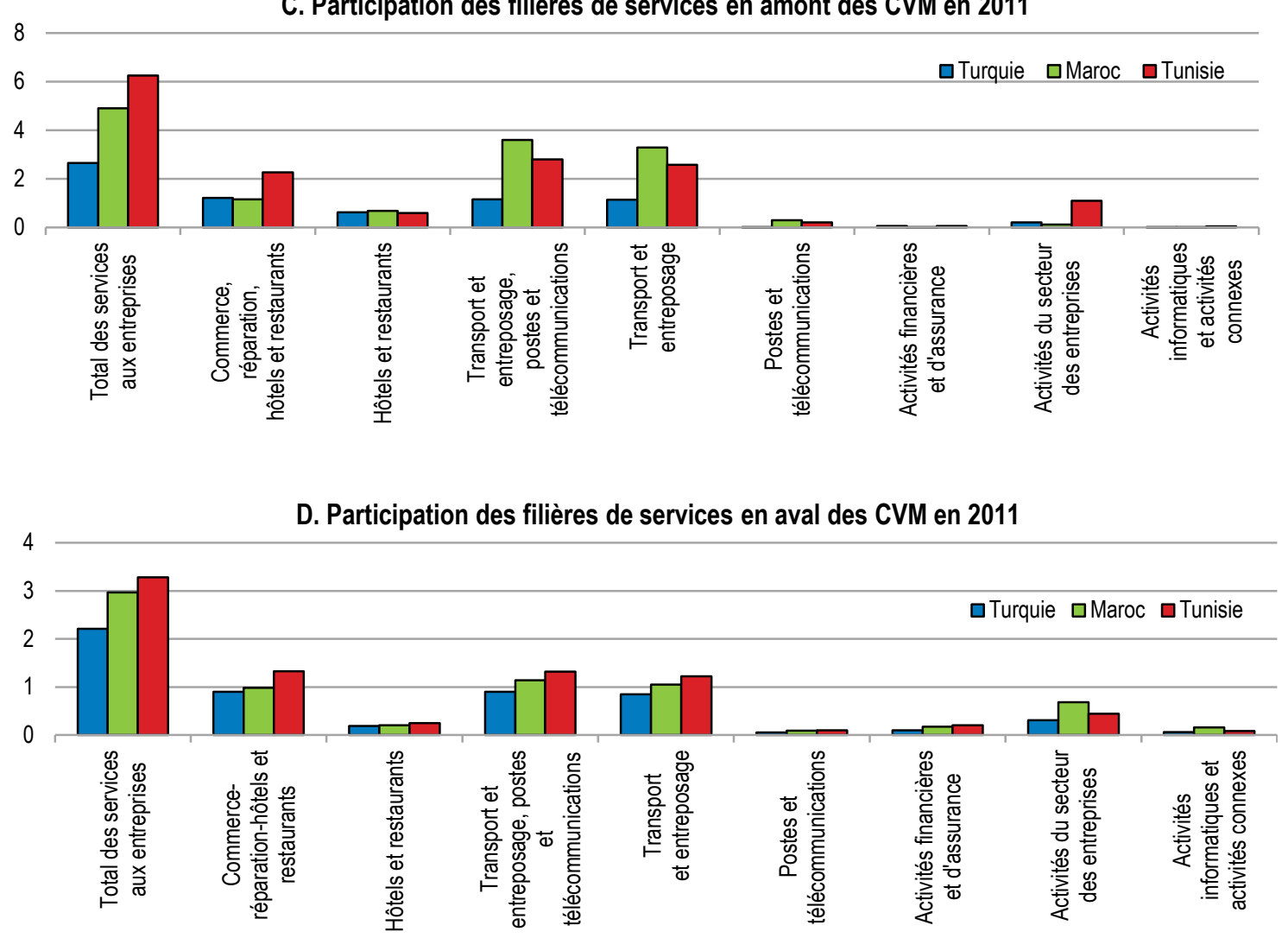

Source: TIVA/ OCDE-2016 ; et calcul des auteurs à partir des données de la base TIVA/ OCDE-2016 